\title{
Influence of the turbulence model for channel flows with strong transverse temperature gradients
}

\author{
Valentin Boutrouche ${ }^{\dagger}$, Erwin Franquet ${ }^{\dagger, \star}$, Sylvain Serra ${ }^{\dagger}$, Rémi Manceau $^{\ddagger,+}$
}

\author{
† Univ. Pau \& Pays Adour, Laboratoire de thermique, énergétique et procédés - IPRA, EA 1932, 64000 Pau, France \\ $\ddagger$ CNRS / Univ. Pau \& Pays Adour, Laboratoire de mathématiques et de leurs applications de Pau - IPRA, UMR 64000, Pau, France and + Inria \\ Bordeaux-Sud-Ouest, project-team CAGIRE \\ * Corresponding author erwin.franquet@univ-pau.fr
}

The effects of a strong transverse temperature gradient on a turbulent Poiseuille flow are studied numerically using Reynoldsaveraged Navier-Stokes (RANS) models. Such a situation is very common for numerous industrial applications. Since a large majority of industrial computations are based on the RANS approach, the aim of the present work is to investigate the ability of different RANS models to reproduce the main physical phenomena at the origin of the asymmetry of the flow and thermal fields. Comparison are performed with available direct numerical simulations (DNS) or large eddy simulation (LES) databases. With the prospect of future application of the models in the industrial context, models based on the widely used eddy-viscosity and simple gradient diffusion (SGDH) hypotheses are compared to more elaborate second-moment closures for the Reynolds stress and turbulent heat flux. The aim is to determine the closure level necessary to reproduce the influence of strong temperature gradients on the turbulent flow, for a wide range of wall-temperature ratios. Eddy-viscosity models prove able to correctly reproduce the asymmetry of the flow and the tendency toward relaminarization close to the hot wall, which are mainly due to the strong variations of the physical properties (namely the molecular viscosity and the density). Discrepancies in the predictions of the different closure levels only appear for the highest temperature ratios. Unfortunately, reliable reference data are lacking for these configurations, which calls for future DNS or refined LES studies.

Keywords: turbulent channel flow, transverse temperature gradient, flow asymmetry, relaminarization, elliptic blending

\section{Contents}

1 Introduction 3

2 Model

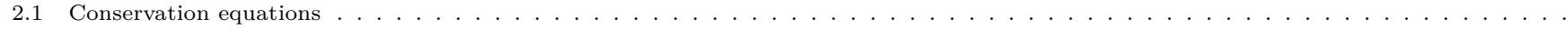

2.2 RANS closure

22.1 EB-BSM

$2.2 .2 k-\varepsilon-v^{2} / k \ldots$

3 Problem description

3.1 Configuration

4 Results

4.1 Isothermal case

4.2 Non-isothermal cases

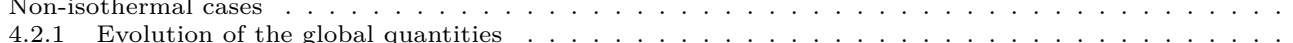

$4.2 .1 \quad$ Evolution of the global quantities . . . . . . . . . . . . . . . . . . . . . . . . . . . . . . . . . . . . . . . . . . . . . . . . . . . .

$4.2 .2 \quad$ Velocity field.

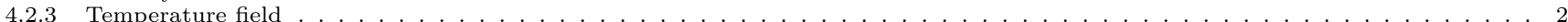

5 Conclusion

A Characteristics of the various cases for each RANS models

C $k-\varepsilon-\overline{v^{2}} / k$ equations

D $k-\omega-$ SST equations

Bibliography 


\section{Nomenclature}

\section{Latin symbols}

$c_{P} \quad$ specific heat capacity, $\mathrm{J} /(\mathrm{kg} \mathrm{K})$

$h$ height, $\mathrm{m}$

$k \quad$ turbulent kinetic energy, $\mathrm{m}^{2} / \mathrm{s}^{2}$

$L \quad$ length, $\mathrm{m}$

$P$ pressure, $\mathrm{Pa}$

Pr Prandtl number, -

$R \quad$ individual ideal perfect gas constant, $\mathrm{J} /(\mathrm{K} \mathrm{kg})$

Re Reynolds number, -

$S_{i j} \quad$ strain tensor, $1 / \mathrm{s}$

$T$ temperature, $\mathrm{K}$

$t \quad$ time, $\mathrm{s}$

$U_{i} \quad i^{\text {th }}$ component of velocity, $\mathrm{m} / \mathrm{s}$

$x_{i} \quad i^{t h}$ component of the position vector, $\mathrm{m}$

$y^{+} \quad$ distance to the wall in wall units, -

\section{Greek symbols}

$\delta_{i j} \quad$ Kronecker symbol, -

$\varepsilon \quad$ turbulent dissipation, $\mathrm{m}^{2} / \mathrm{s}^{3}$

$\lambda$ thermal conductivity, $\mathrm{W} /(\mathrm{K} \mathrm{m})$

$\mu \quad$ dynamic viscosity, $\mathrm{kg} /(\mathrm{m} \mathrm{s})$

$\nu \quad$ kinematic viscosity, $1 /\left(\mathrm{m}^{2} \mathrm{~s}\right)$

$\rho \quad$ density, $\mathrm{kg} / \mathrm{m}^{3}$

$\tau \quad$ viscous tensor, $\mathrm{Pa}$

$\omega \quad$ specific dissipation rate, $1 / \mathrm{s}$

\section{Subscripts and superscripts}

1 cold wall

2 hot wall

$\bar{X} \quad$ Favre average of the variable $X$

$X^{\prime} \quad$ Fluctuation of the variable $X$

$b \quad$ bulk

$h$ homogeneous

$m$ mean

$r \quad$ ratio

$t \quad$ turbulent

$w \quad$ wall

\section{Acronyms}

DFM differential flux model

DNS direct numerical simulation

EB elliptic blending

GGDH generalized gradient diffusion hypothesis

LES

RANS Reynolds-averaged Navier-Stokes

RSM Reynolds-stress model

SGDH simple gradient diffusion hypothesis

SSG Speziale-Sarkar-Gatski

SST shear stress transport 


\section{Introduction}

A better understanding and modeling of the physical mechanisms observed in turbulent flows with strong temperature gradients are important for many industrial processes, in particular for the design of high temperature solar receiver for concentrated solar power plants. These flows are characterized by strong variations of the fluid properties, such that, even in the forced convection regime, they significantly deviate from isothermal flows, with a possible tendency to relaminarize, which can significantly reduce heat transfer [1].

Since the Mach number is very low in such applications, the problem can be treated using the low-Mach-number approximation [2], which filters out the acoustics but preserves the variation of density with temperature. In order to investigate the performance, for variable property flows, of RANS models, which are the workhorses for industrial applications, the present paper focuses on turbulent channel flows with strong temperature gradients. A limited number of reference databases are available in the literature: indeed, most of the direct numerical simulation (DNS) studies of turbulent channel flows performed since the pioneering work of Kim et al. [3] are either isothermal [4] 6], with temperature as a passive scalar $7-12$ or include buoyancy effects 13 21], but not the variation of molecular viscosity and conductivity with temperature. Nicoud \& Poinsot [22] published the first computations with variable physical properties, for two different temperature ratios $T_{r}=T_{2} / T_{1}=1.01$ and 2 at the friction Reynolds number $\operatorname{Re}_{\tau m}=180$. Debusschere \& Rutland 23] computed both turbulent Couette and Poiseuille flows, with $\operatorname{Re}_{\tau m}=160$ and 180, respectively, with imposed wall temperatures but only for $T_{r}=1.01$. Eventually, Auléry et al. [24 extended the work of Nicoud \& Poinsot to turbulent Poiseuille flows at $\operatorname{Re}_{\tau m}=180$ and 395 and for $T_{r}=1$ and 2 . Very recently, Patel et al. 25, 26. performed similar computations for a different configuration, where the flow is internally heated.

At the same time, limitations due to the cost of DNS have led some authors to perform similar studies with LES: Wang \& Fletcher 27 studied a non-isothermal channel flow with the temperature ratio $T_{r}=3$ for $\operatorname{Re}_{\tau m}=180$; Lessani \& Papalexandris $\left[28\right.$ extended this work up to $T_{r}=9$; Serra et al. $\left[29\right.$ covered a range temperature ratios $T_{r}=1,1.01$, 1.07, 2 and 5, for $\operatorname{Re}_{\tau m}=180$ and 395. This work has been extended to higher friction Reynolds number by [30] but only up to $T_{r}=3$. An overview of available results is given in table 1

Table 1 - Available simulations for non-isothermal turbulent flows in plane channels

\begin{tabular}{|c|c|c|c|c|c|c|c|c|c|c|c|}
\hline$T_{r}$ & & 1 & 1.01 & 1.02 & 1.07 & 2 & 3 & 5 & 6 & 8 & 9 \\
\hline \multirow{2}{*}{$\operatorname{Re}_{\tau m}=180$} & DNS & 39 & 22,23 & & & \begin{tabular}{|l|l|}
22 & 24 \\
\end{tabular} & & & & & \\
\hline & LES & {$[29,31,32$} & \begin{tabular}{|l|l|}
28 & 33 \\
\end{tabular} & 27 & & \begin{tabular}{|l|l|l|}
28 & 30 & 32
\end{tabular} & 27,30 & \begin{tabular}{|l|l|}
29 & 30 \\
\end{tabular} & 28 & 28 & 28 \\
\hline \multirow{2}{*}{$\operatorname{Re}_{\tau m}=395$} & DNS & \begin{tabular}{l|l|l}
3 & 9 & 24 \\
\end{tabular} & & & & $\mid \overline{24}$ & & & & & \\
\hline & LES & \begin{tabular}{|l|l|}
29 & 32 \\
\end{tabular} & 30 & & 29,32 & \begin{tabular}{|l|l|}
29 & 30, \\
\end{tabular} & & 29 & & & \\
\hline
\end{tabular}

Accounting for the variations with temperature of the molecular viscosity and conductivity of the fluid is necessary in the presence of strong temperature differences. In particular, many industrial applications of RANS models make use of Sutherland's law [34] and similar relations for the conductivity, or tabulated values (e.g. 18 35 37]). A large majority of these studies are concerned with flows in the vertical direction, with combined effects of buoyancy and variable properties of the fluid. In the configuration of strongly heated horizontal channel flows that are representative of the geometry of solar receivers, buoyancy does not affect the flow since the Richardson number is lower than $10^{-2}$, and there is, to our knowledge, no RANS study of the appearance of asymmetric turbulent field due to variable properties of the fluid.

Consequently, the aim of the present work is to investigate the performance of different levels of turbulence closure, from simple eddy-viscosity and diffusivity hypotheses to sophisticate second-moment closures for the Reynolds stress and the turbulent heat flux. The performance indicator is the ability to reproduce the main physical phenomena at the origin of the asymmetry of the mean flow and the turbulent statistics in channel flows with strong temperature gradients. The equations of motion are written in the low-Mach-number approximation, with temperature-dependent physical properties of the fluid (density, viscosity and thermal conductivity). Since the wall layer is strongly modified, due to these variations of the physical properties, wall functions are not valid, such that near-wall turbulence models are selected. In order to compare the merits of different approaches, the widely used $k-\omega-$ SST model [38] is compared to an eddy-viscosity model of the elliptic relaxation/blending family, the $k-\varepsilon-\overline{v^{2}} / k$ model [39], both associated to the simple gradient diffusion hypothesis (SGDH) for the turbulent heat flux; and to the Elliptic Blending Reynolds-stress Model (EB-RSM) [40, 41], associated with the SGDH, the generalized gradient diffusion hypothesis (GGDH) or the differential flux model (DFM).

The paper is organized as follows: in section 2 the system of partial differential equations of the low-Mach-number approximation is recalled, with the associated variable physical properties, and the turbulence models are introduced. The geometry of the problem and numerical setup are presented in section 3 . Section 4 is dedicated to the presentation of the results, and conclusions are drawn in Section 5 


\section{$2 \quad$ Model}

\subsection{Conservation equations}

For flows under consideration, the fluid can be treated as a non-weighting Newtonian fluid with temperature-dependent physical properties. The use of density-weighted (Favre) averaging to decompose the turbulent flow into mean and fluctuating motions simplifies the equations by avoiding the appearance of density fluctuations. Owing to the very low value of the Mach number in the targeted applications, the low-Mach-number approximation can be used, such that pressure is decomposed into hydrodynamic $P$ and thermodynamic $P_{0}$ components 2 . DNS and LES databases used as references in the present paper were computed using this approximation [22 24 29]. In the turbulent regime, the variables are decomposed using Favre averaging - and it is standard to neglect in the RANS equations the influence of the turbulent fluctuations of molecular viscosity and thermal conductivity [42], which leads to

$$
\begin{aligned}
\frac{\partial \rho}{\partial t}+\frac{\partial \rho \overline{U_{i}}}{\partial x_{i}} & =0 \\
\rho \frac{\partial \overline{U_{i}}}{\partial t}+\rho \overline{U_{j}} \frac{\partial \overline{U_{i}}}{\partial x_{j}} & =-\frac{\partial \bar{P}}{\partial x_{i}}+\frac{\partial}{\partial x_{j}}\left[\mu\left(\frac{\partial \overline{U_{i}}}{\partial x_{j}}+\frac{\partial \overline{U_{j}}}{\partial x_{i}}\right)\right]-\frac{2}{3} \frac{\partial}{\partial x_{j}}\left(\mu \frac{\partial \overline{U_{j}}}{\partial x_{j}}\right)-\frac{\partial \rho \overline{U_{i}^{\prime} U_{j}^{\prime}}}{\partial x_{j}}, \\
\rho c_{p} \frac{\partial \bar{T}}{\partial t}+\rho c_{p} \overline{U_{i}} \frac{\partial \bar{T}}{\partial x_{i}} & =\frac{\partial \overline{P_{0}}}{\partial t}+\overline{U_{i}} \frac{\partial \overline{P_{0}}}{\partial x_{i}}+\frac{\partial}{\partial x_{i}}\left(\lambda \frac{\partial \bar{T}}{\partial x_{j}}\right)-\frac{\partial}{\partial x_{i}}\left(\rho c_{p} \overline{U_{i}^{\prime} T^{\prime}}\right),
\end{aligned}
$$

associated to the ideal perfect gas law for air,

$$
\overline{P_{0}}=\rho R \bar{T} .
$$

$\rho, \mu$ and $\lambda$ denote the Reynolds-averaged density, dynamic viscosity and thermal conductivity, respectively; $U_{i}$ denotes the components of velocity and $T$ the temperature, and $U_{i}^{\prime}$ and $T^{\prime}$ their respective fluctuations in the Favre decomposition. $c_{p}$ stands for the heat capacity at constant pressure.

A distinctive feature of the present study, necessary to reproduce the physical phenomena observed with strong temperature gradients, is that the mean dynamic viscosity and thermal conductivity are dependent on the mean temperature. In accordance with existing databases [22,24 29], Sutherland's law, valid between $220 \mathrm{~K}$ and $1900 \mathrm{~K}$, is assumed, and the heat capacity and the Prandtl number are considered constant, such that

$$
\begin{aligned}
\mu(T) & =1.461 \cdot 10^{-6} \frac{T^{1.5}}{T+111}, \\
\lambda(T) & =\frac{\mu(T) c_{p}}{\operatorname{Pr}}=\frac{1.468 \cdot 10^{-3}}{\operatorname{Pr}} \frac{T^{1.5}}{T+111} .
\end{aligned}
$$

\subsection{RANS closure}

Most of the industrial applications of the RANS approach make use of wall functions to avoid the resolution of the nearwall region. Nonetheless, in the cases under consideration herein, due to the temperature gradient and the related strong variations of the physical properties, turbulence does not comply with the standard law of the wall [24]29], such that the recourse to wall functions must be avoided.

Among numerous models valid in the near-wall region, the $k-\omega$-SST model [38] is one of the most widely used, such that it is selected here for comparison purposes. The most recent version of this standard model can be found in [43] and are not repeated here. However, for heat transfer prediction, the satisfaction of the two-component limit of turbulence is crucial, and the models derived from the elliptic relaxation concept of Durbin [44] have been successfully applied to many configurations (for instance, $[39,45,48]$ ). The elliptic relaxation approach enforces the correct asymptotic behavior in the vicinity of the wall via the resolution of six additional equations for the pressure-strain correlation in second-moment closures. In order to reduce the number of equations and circumvent the numerical stiffness of the model, Manceau \& Hanjalić 40 have introduced the elliptic blending strategy. In the present study, two models based on this approach are selected:

- The Elliptic Blending Reynolds-stress model (EB-RSM) 41.

- The $k-\varepsilon-\overline{v^{2}} / k$ eddy-viscosity model proposed by Billard \& Laurence [39, which is an eddy-viscosity model derived from the EB-RSM. 


\subsubsection{EB-RSM}

The EB-RSM is a second-moment closure derived from Durbin's elliptic relaxation approach [44, 49], which permits to migrate from a far-field model, the SSG Reynolds-stress model [50], to a near-wall model, which satisfies the asymptotic behavior of the redistribution term. The transport equations of the model write

$$
\begin{aligned}
\frac{\mathrm{d} \rho \overline{U_{i}^{\prime} U_{j}^{\prime}}}{\mathrm{d} t} & =\rho \mathcal{P}_{i j}+\rho \phi_{i j}-\rho \varepsilon_{i j}+D_{i j}^{\mu}+D_{i j}^{T}, \\
\frac{\mathrm{d} \rho \varepsilon}{\mathrm{d} t} & =\frac{C_{\varepsilon 1}^{\prime} \rho \mathcal{P}-C_{\varepsilon 2} \rho \varepsilon}{\mathbb{T}}+\frac{\partial}{\partial x_{k}}\left(\frac{C_{\mu}}{\sigma_{\varepsilon}} \rho \tau_{k l} \mathbb{T} \frac{\partial \varepsilon}{\partial x_{l}}\right)+\frac{\partial}{\partial x_{k}}\left(\mu \frac{\partial \varepsilon}{\partial x_{k}}\right), \\
\alpha-\mathcal{L}^{2} \nabla^{2} \alpha & =1 .
\end{aligned}
$$

The far-field $(h)$ and near-wall $(w)$ models for $\phi_{i j}-\varepsilon_{i j}$ are activated through the blending formula

$$
\phi_{i j}-\varepsilon_{i j}=\left(1-\alpha^{3}\right)\left(\phi_{i j}^{w}-\varepsilon_{i j}^{w}\right)+\alpha^{3}\left(\phi_{i j}^{h}-\varepsilon_{i j}^{h}\right)
$$

in the region far from the wall $(\alpha \rightarrow 1)$ and close to the wall $(\alpha \rightarrow 0)$, respectively. Complementary equations and values for the various constants are given in appendix $B$

With such a model, since all the components of the Reynolds-stress tensor are accessible, several options are available to express the turbulent heat flux appearing in the energy equation (2.1c). In the present study, three closures are considered:

- The Simple Gradient Diffusion Hypothesis (SGDH)

$$
\rho \overline{U_{i}^{\prime} T^{\prime}}=-\frac{\mu_{t}}{\operatorname{Pr}_{t}} \frac{\partial \bar{T}}{\partial x_{i}}
$$

with $\mu_{t}=C_{\mu} \rho k^{2} / \varepsilon$ and $C_{\mu}=0.09, k=\overline{U_{i}^{\prime} U_{i}^{\prime}} / 2$ and $\operatorname{Pr}_{t}=1$, which is used here as a reference since most of the commercial CFD codes rely on this hypothesis, even for Reynolds-stress models.

- The Generalized Gradient Diffusion Hypothesis (GGDH), which introduces an anisotropic diffusion coefficient through the Daly-Harlow relation [51]:

$$
\rho \overline{U_{i}^{\prime} T^{\prime}}=-C_{\theta} \rho \frac{k}{\varepsilon} \overline{U_{i}^{\prime} U_{j}^{\prime}} \frac{\partial \bar{T}}{\partial x_{j}},
$$

with $C_{\theta}=0.22$.

- A Differential Flux Model (DFM) [52], which solves a transport equation for the turbulent heat flux

$$
\frac{\mathrm{d} \rho \overline{U_{i}^{\prime} T^{\prime}}}{\mathrm{d} t}=\rho \mathcal{P}_{i \theta}^{U}+\rho \mathcal{P}_{i \theta}^{T}+\rho \mathcal{G}_{i \theta}+\rho \phi_{i \theta}^{h}-\rho \varepsilon_{i \theta}^{h}+D_{i \theta}^{T}+D_{i \theta}^{\mu} .
$$

Indeed, one of the objectives of the present study is to evaluate the potential benefit of using a full second-moment closure (for both the Reynolds-stress and the turbulent heat flux), compared to using a simple algebraic heat flux model in association with a Reynolds-stress model.

2.2.2 $k-\varepsilon-\overline{v^{2}} / k$

The $k-\varepsilon-\overline{v^{2}} / k$ model 39$]$ is an eddy-viscosity model, based on the standard Boussinesq relation

$$
\rho \overline{U_{i}^{\prime} U_{j}^{\prime}}=-\mu_{t}\left(\frac{\partial \overline{U_{i}}}{\partial x_{j}}+\frac{\partial \overline{U_{j}}}{\partial x_{i}}-\frac{2}{3} \frac{\partial \overline{U_{k}}}{\partial x_{k}} \delta_{i j}\right)+\frac{2}{3} \rho k \delta_{i j} .
$$

The main particularity of this model is that the turbulent viscosity $\mu_{t}$ is modeled as

$$
\begin{aligned}
\mu_{t} & =C_{\mu} \rho \varphi k \min \left(\mathbb{T}, \mathbb{T}_{\lim }\right), \\
\mathbb{T} & =\sqrt{\left(\frac{k}{\varepsilon}\right)^{2}+C_{T}^{2} \frac{\nu}{\varepsilon}}, \\
\mathbb{T}_{\lim } & =\frac{0.6}{\sqrt{6} C_{\mu} \varphi \sqrt{\underline{\underline{S}:}} \underline{\underline{S}}},
\end{aligned}
$$


in which a new scale $\varphi=\overline{v^{2}} / k$ is introduced, such that $\varphi k$ characterizes the energy of the wall normal turbulent fluctuations. Similar to the EB-RSM, the redistribution in the $\varphi$ equation is modeled using an elliptic blending of near-wall $\left(f_{w}\right)$ and far-field $\left(f_{h}\right)$ terms involving the parameter $\alpha$ that makes the model implicitly sensitive to the distance to the wall:

$$
\begin{aligned}
\frac{\mathrm{d} \rho k}{\mathrm{~d} t} & =\rho \mathcal{P}-\rho \varepsilon+\frac{\partial}{\partial x_{i}}\left[\left(\frac{\mu}{2}+\frac{\mu_{t}}{\sigma_{k}}\right) \frac{\partial k}{\partial x_{i}}\right]-C_{\varepsilon 3}(1-\alpha)^{3} \rho \frac{k}{\varepsilon} 2 \nu \nu_{t}\left(\partial_{k} \partial_{j} \overline{U_{i}}\right)\left(\partial_{k} \partial_{j} \overline{U_{i}}\right), \\
\frac{\mathrm{d} \rho \varepsilon}{\mathrm{d} t} & =\frac{C_{\varepsilon 1} \rho \mathcal{P}-C_{\varepsilon 2}^{*} \rho \varepsilon}{\mathbb{T}}+\frac{\partial}{\partial x_{i}}\left[\left(\frac{\mu}{2}+\frac{\mu_{t}}{\sigma_{k}}\right) \frac{\partial k}{\partial x_{i}}\right], \\
\frac{\mathrm{d} \varphi}{\mathrm{d} t} & =\left(1-\alpha^{3}\right) f_{w}+\alpha^{3} f_{h}-\mathcal{P} \frac{\varphi}{k}+\frac{2}{k} \frac{\nu_{t}}{\sigma_{k}} \frac{\partial \varphi}{\partial x_{i}} \frac{\partial k}{\partial x_{i}}+\frac{\partial}{\partial x_{i}}\left[\left(\frac{\nu}{2}+\frac{\nu_{t}}{\sigma_{\varphi}}\right) \frac{\partial \varphi}{\partial x_{i}}\right], \\
\alpha-\mathcal{L}^{2} \nabla^{2} \alpha & =1 .
\end{aligned}
$$

Note that, here, $\varepsilon$ is defined as the homogeneous part of the dissipation rate, following Jakirlić \& Hanjalić [53]. This explains the presence of the factor $1 / 2$ in the molecular diffusion term.

Further details and coefficients are available in appendix $\mathrm{C}$

\section{Problem description}

\subsection{Configuration}

A turbulent Poiseuille flow in a bi-periodic plane channel with a (possibly strong) temperature gradient is considered, as depicted in figure 1

The following parameters are introduced, in accordance with the available literature:

$$
\begin{aligned}
& \operatorname{Re}_{\tau m}=\frac{\operatorname{Re}_{\tau, 1}+\operatorname{Re}_{\tau, 2}}{2} \\
& \operatorname{Re}_{\tau, 1}=\frac{\rho_{w, 1} U_{\tau, 1} h}{\mu_{w, 1}} \\
& \operatorname{Re}_{\tau, 2}=\frac{\rho_{w, 2} U_{\tau, 2} h}{\mu_{w, 2}} \\
& \operatorname{Re}_{b}=\frac{\rho_{b} U_{b} h}{\mu_{b}} \\
& U_{b}=\frac{1}{2 h} \int \bar{U}(y) d y \\
& T_{m}=\frac{1}{2 h} \int \bar{T}(y) d y \\
& T_{r}=\frac{T_{2}}{T_{1}} \\
& U^{+}=\frac{\bar{U}}{U_{\tau}} \\
& U_{\tau}=\sqrt{\frac{\tau_{w}}{\rho_{w}}} \\
& \tau_{w}=\left.\mu_{w} \frac{d \bar{U}}{d y}\right|_{w} \\
& T^{+}=\frac{\bar{T}-T_{w}}{T_{\tau}} \\
& T_{\tau}=\frac{d \bar{T}}{\rho_{w} c_{p} U_{\tau}} \\
& \lambda_{w} \\
& \text { ing }
\end{aligned}
$$

with $\operatorname{Re}_{\tau}$ the friction Reynolds number based on the channel half-height $h, \rho_{b}$ and $\mu_{b}$ the bulk density and dynamic viscosity, $\rho_{w}, \mu_{w}$ and $\lambda_{w}$ the density, dynamic viscosity and thermal conductivity at the wall, respectively. The indices 1 and ${ }_{2}$ indicate if the term is computed at the cold (1) or hot (2) wall. 

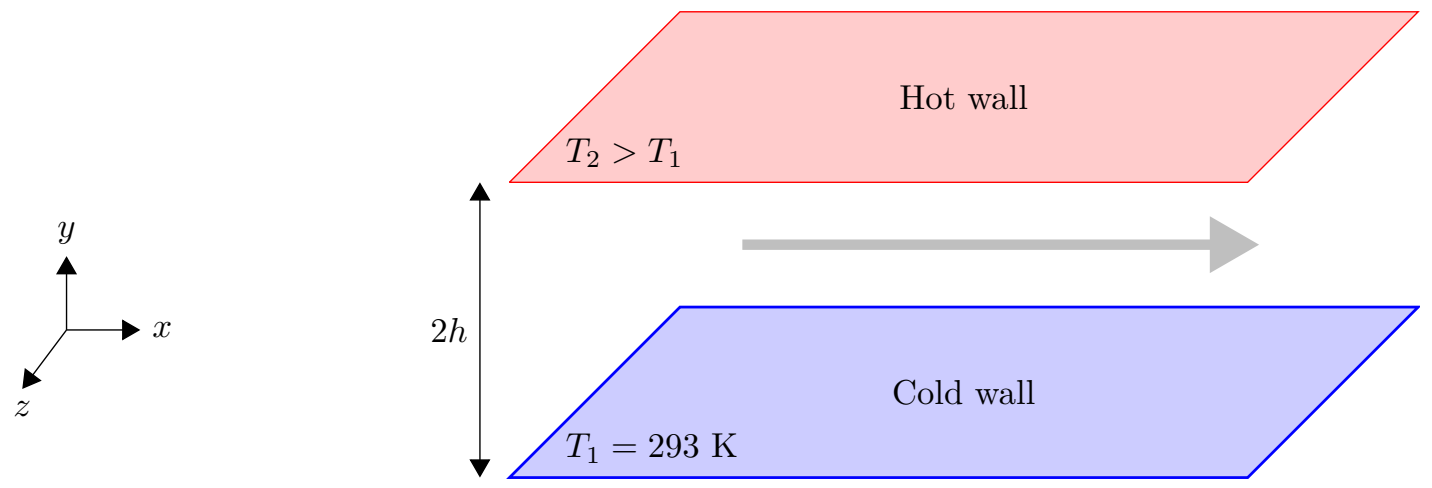

Figure 1 - Description of the studied configuration

The periodicity hypothesis in the streamwise direction requires an additional source term in the right-hand side of the momentum equation 2.1b), to account for the constant streamwise pressure gradient,

$$
\beta=-\frac{\partial P}{\partial x}=\frac{\tau_{w, 1}+\tau_{w, 2}}{2 h},
$$

which is adapted all along the computation to target a given $\operatorname{Re}_{\tau m}$.

The half-height of the channel $h$ is $0.15 \mathrm{~m}$. Two mean friction Reynolds numbers $\operatorname{Re}_{\tau m}=180$ and 395 and five temperature ratios $T_{r}=1,1.01,1.07,2$ and 5 are considered in the present study.

\subsection{Numerical method}

The open-source CFD code Code_Saturne [54], based on the finite volume method, has been used to perform the present calculations. Concerning the spatial discretization, a second order linear upwind (SOLU) scheme is applied for the convective terms and an iterative reconstruction is used, together with an arithmetic interpolation, for the diffusive and gradients terms. Finally, the pressure-velocity coupling is based on the standard SIMPLEC algorithm and the temporal integration is performed with the implicit Euler method.

The models under consideration require a cell center located at $y^{+} \approx 1$ to correctly describe the viscous sublayer. As shown in figure 2, in order to account for the expected unequal friction velocity between the two walls, an asymmetrical mesh is generated in three steps, for each side: i) a first zone (a) is composed of regular cells contained in the low-Reynolds number region $\left(y^{+}<20\right)$, ii) a second zone (b) involves irregular cells whose characteristic size obeys a geometrical expansion, iii) a third zone (c) contains regular cells that connect the two partial meshes extruded from both sides.

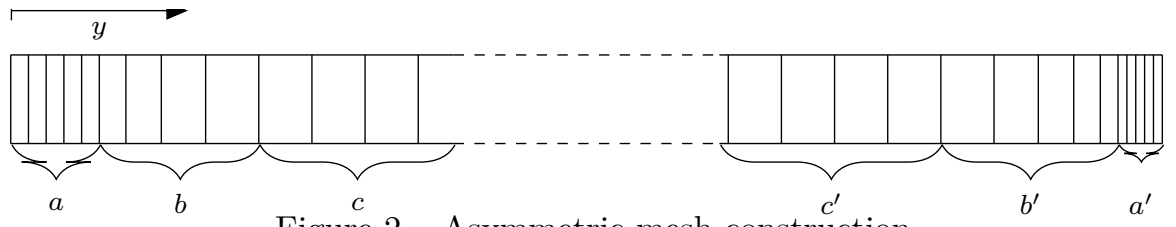

Figure 2 - Asymmetric mesh construction

\section{Results}

Since the aim of the paper is to evaluate the capability of different RANS models, using either first-moment or secondmoment closures for the Reynolds stress and the turbulent heat flux, to reproduce the effects due to a transverse temperature gradient in a channel flow, comparisons are made with the few DNS databases available in the literature, which are summarized in Table 2. However, our purpose is to investigate the response of different RANS models over a wide range of parameters, with wall-temperature ratios up to 5, for which DNS data are missing. Since Serra et al. [29] have performed a systematic LES study for the two Reynolds numbers $\operatorname{Re}_{\tau, m}=180$ and 395, with temperature ratios ranging from 1 (isothermal) to 5, their LES database will be used for comparison purposes, although it will become clear in the next section that these computations are under-resolved, such that they will only provide qualitative indications. 
Table 2 - Presentation of the selected references, DNS $(\checkmark)$ and LES (\%), used for RANS results benchmarking

\begin{tabular}{|c|c|c|c|c|c|c|c|c|}
\hline References & $180-1$ & $180-1.01$ & $180-2$ & $180-5$ & $395-1$ & $395-1.07$ & $395-2$ & $395-5$ \\
\hline Kim et al. [3] & $\checkmark$ & & & & & & & \\
\hline Moser et al. 4 & & & & & $\checkmark$ & & & \\
\hline Nicoud \& Poinsot 22 & & $\checkmark$ & $\checkmark$ & & & & & \\
\hline Serra et al. 29 & $\%$ & $\%$ & $\%$ & $\%$ & $\%$ & $\%$ & $\%$ & $\%$ \\
\hline Auléry et al. 24 & & & $\checkmark$ & & & & $\checkmark$ & \\
\hline
\end{tabular}

\subsection{Isothermal case}

For the isothermal case (temperature ratio $T_{r}=1$ ), the physical properties of the fluid are constant and the flow is symmetric. The comparison of DNS, LES and RANS results makes possible an initial evaluation of the accuracy of the different models in this standard channel flow configuration. In figure 3, the longitudinal velocity is plotted, nondimensionalized by either the maximum velocity or the friction velocity, along the transverse direction. The profiles, in particular when plotted in wall units, exhibit significant discrepancies with the DNS results. All the RANS models correctly reproduce the flow at $\operatorname{Re}_{\tau}=180$, but the $k-\varepsilon-\overline{v^{2}} / k$ under-predicts the mean velocity at $\operatorname{Re}_{\tau}=395$. The most striking feature is the significant over-prediction of the flow rate by the LES, in particular for the higher Reynolds number, which is due to the under-resolution in the transverse direction 29, section "Validation", p. 513]. It is therefore worth mentioning that comparisons with LES will mainly focus on the qualitative evolution of the flow features with the temperature gradient and not on the quantitative values of the variables.

\subsection{Non-isothermal cases}

As mentioned above, two friction Reynolds numbers and five temperature ratios are investigated. Before examining the mean velocity, mean temperature and turbulent fields in sections 4.2 .2 and 4.2 .3 section 4.2 .1 provides an overview of the evolution with the temperature ratio of the bulk quantities and the friction Reynolds numbers at the two walls. The corresponding values obtained using RANS models are given in Appendix A for all cases in tables 3 to 7 , in comparison with values given by DNS $[22]$ and LES $[29]$ simulations (in blue and red, respectively), when available.

\subsubsection{Evolution of the global quantities}

The bulk Reynolds number and velocity as a function of the temperature ratio $T_{r}$ are first shown in figures 4 and 5 As expected [28], the bulk Reynolds number decreases with the temperature ratio, and the different models, including LES, give very similar results, for both values of the averaged friction Reynolds number $\operatorname{Re}_{\tau m}$. In contrast, the bulk velocity grows with the temperature ratio, which means that the other term involved in the Reynolds number definition, that is to say the term $\frac{\mu_{b}}{\rho_{b}}$ is strongly increased, as can be seen in figure 6 Moreover, it can be seen that large discrepancies appear for the prediction of $U_{b}$ between the first-moment and second-moment closures, as well as with the available LES results, for large values of $T_{r}$. However, the results are less scattered for the bulk Reynolds number than for the bulk velocity, which suggests that the dynamics of the mean flow is mainly governed by the evolution of $\mu / \rho$ with $T_{r}$. It is noted that for the present fully-developed flows, the left hand sides of the mean conservation equations $(2.1 \mathrm{~b})$ and $(2.1 \mathrm{c})$ are zero, such that the ratio $\mu / \rho$ is mainly related to the relative weights of the viscous and turbulent transport terms in the equations.

The evolution of the cold and hot side friction Reynolds numbers, i.e. for the lower and upper walls, is now considered. As shown in figure 7 the two friction Reynolds numbers take rather different values when the temperature gradient increases, which is still in conformity with previous observations $24,28,29,55$. Moreover, it is worth noticing that for the highest temperature ratios, the friction Reynolds number for the hot wall can become very low, which suggests a tendency towards a laminar flow $29,56,57$. Eventually, in figure 8 the mean temperatures are plotted for the two friction Reynolds numbers. The same graph also shows the linear evolution of the mean temperature that would be observed for a flow with constant physical properties, whose profile would be symmetrical. In the case of variable properties, the behavior is very different in laminar and turbulent regimes. For laminar flows, since the thermal conductivity increases with the temperature, the mean temperature should be above this line in order to maintain a constant heat flux, in order to satisfy the energy balance. However for turbulent flows, the trend is inverted [3.22, 24], as predicted by the RANS models (see section 4.2.3 and contrary to LES. Concerning the RANS results, a significant discrepancy between the models can be observed, especially for the lower friction Reynolds number. 

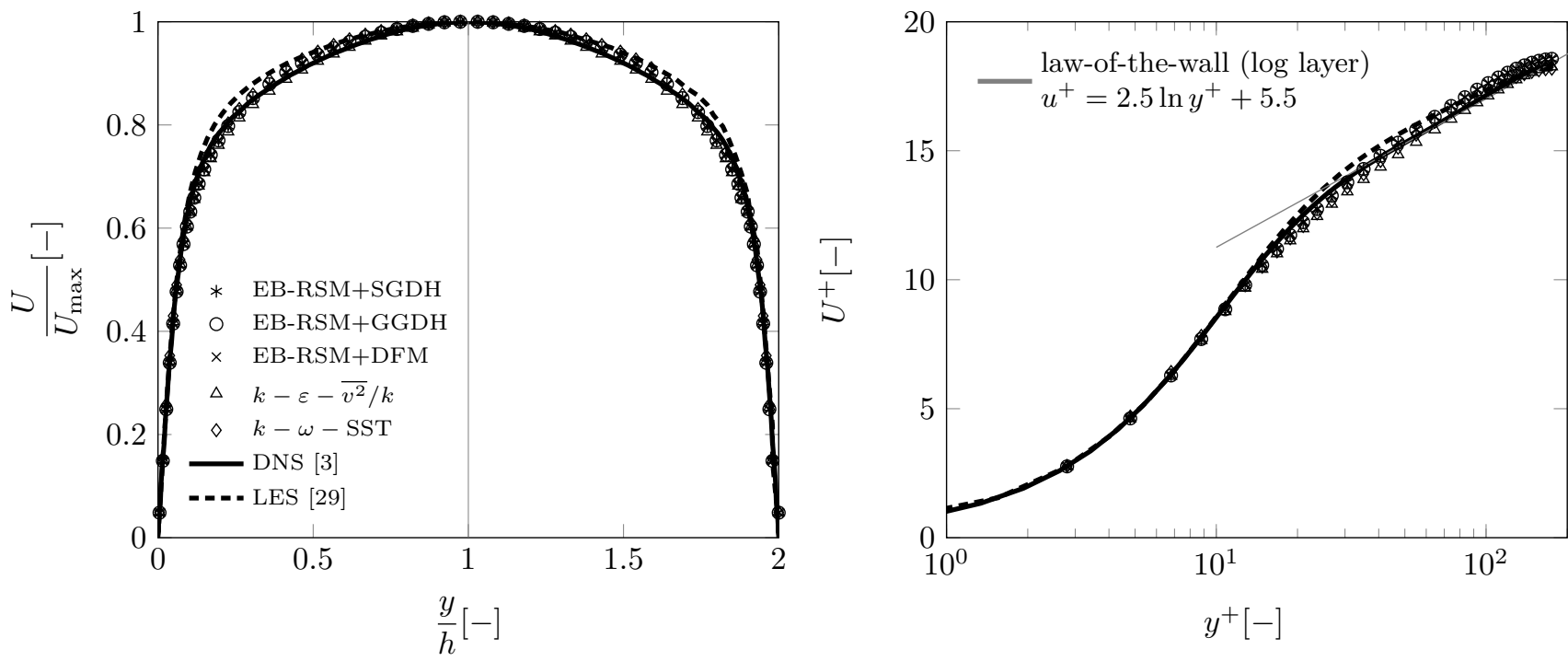

(a) $\operatorname{Re}_{\tau}=180$
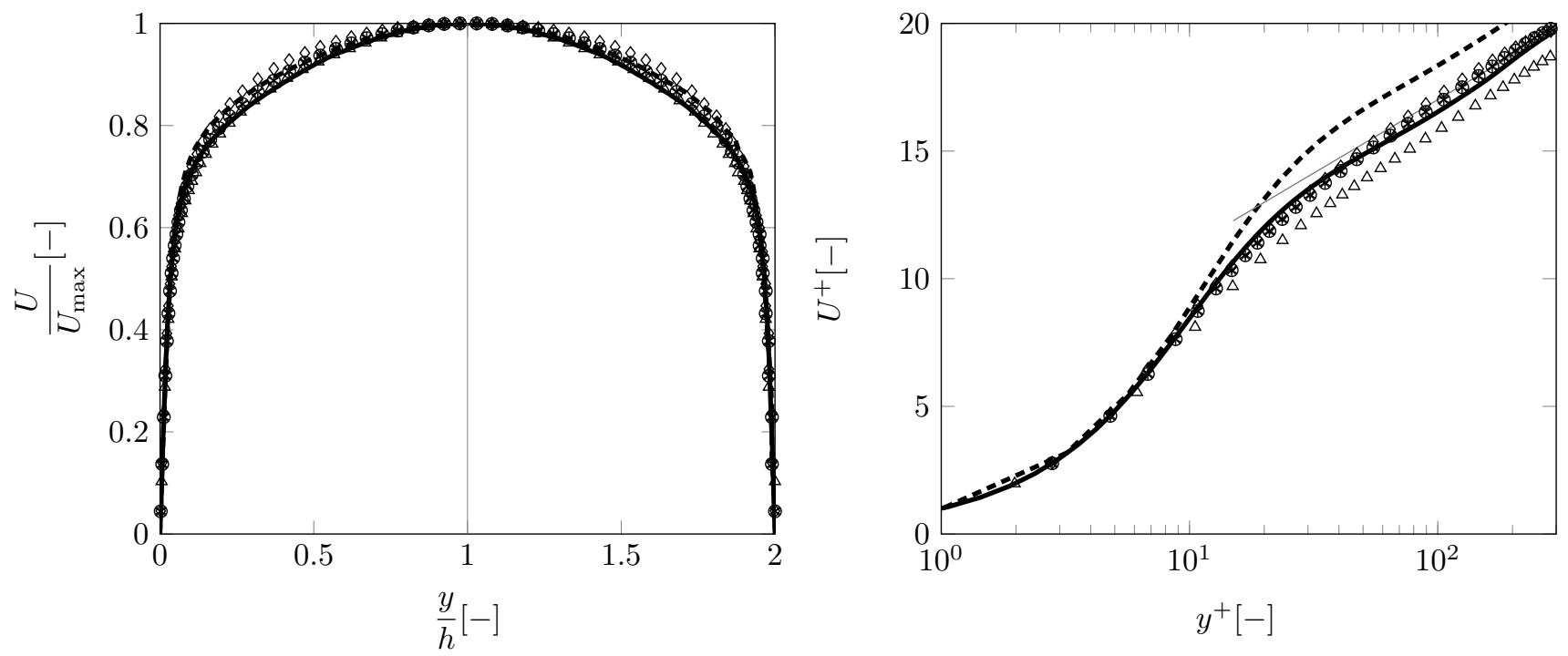

(b) $\operatorname{Re}_{\tau}=395$

Figure 3 - Non-dimensional longitudinal velocity for isothermal flows $\left(T_{r}=1\right)$ 


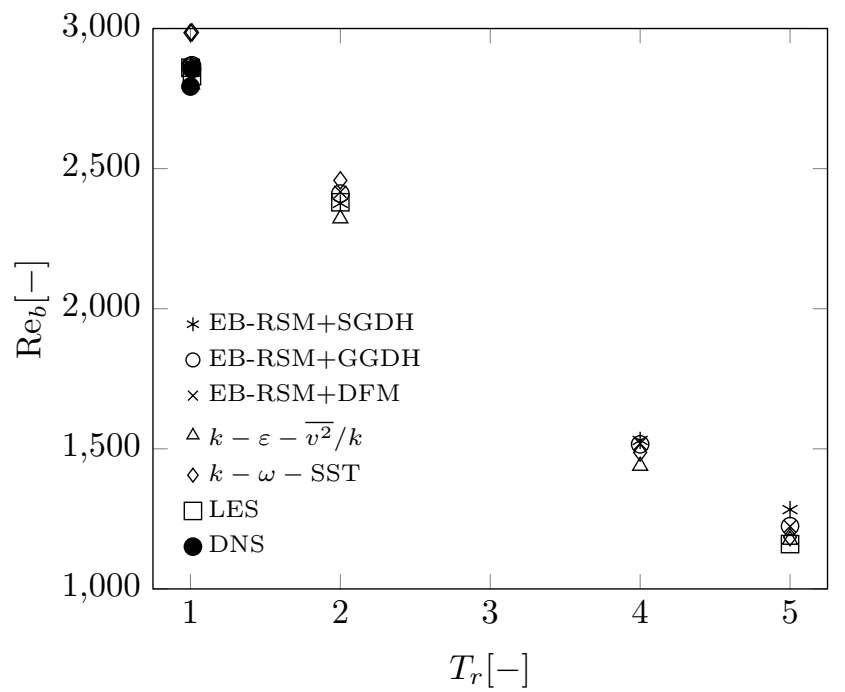

(a) $\operatorname{Re}_{\tau m}=180$

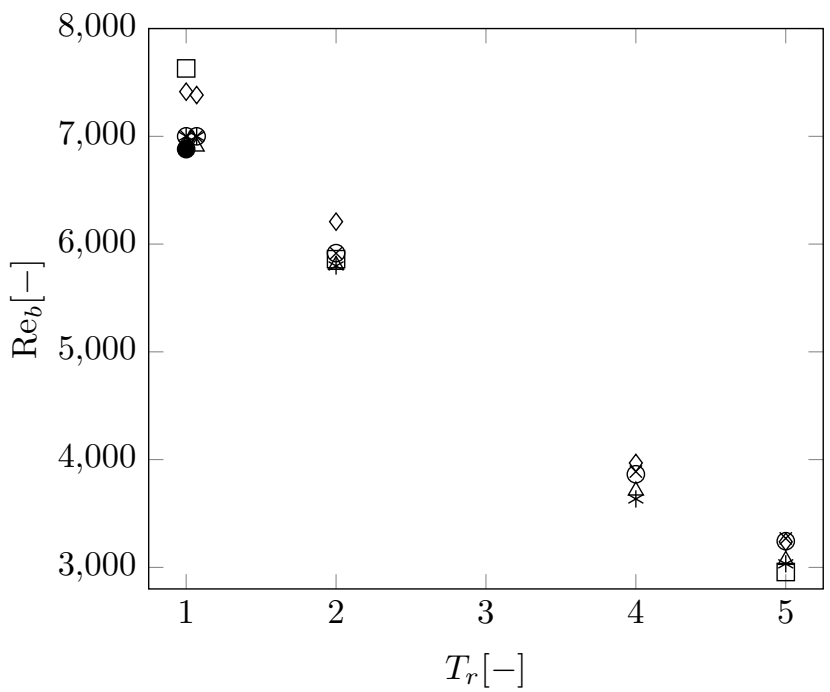

(b) $\operatorname{Re}_{\tau m}=395$

Figure 4 - Evolution of the bulk Reynolds number as a function of the temperature ratio $T_{r}$

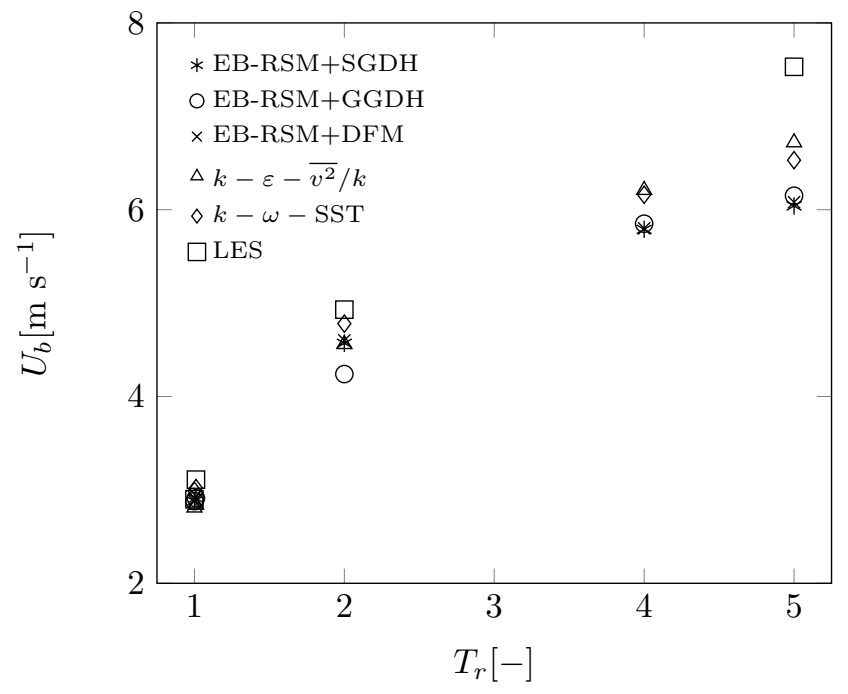

(a) $\operatorname{Re}_{\tau m}=180$

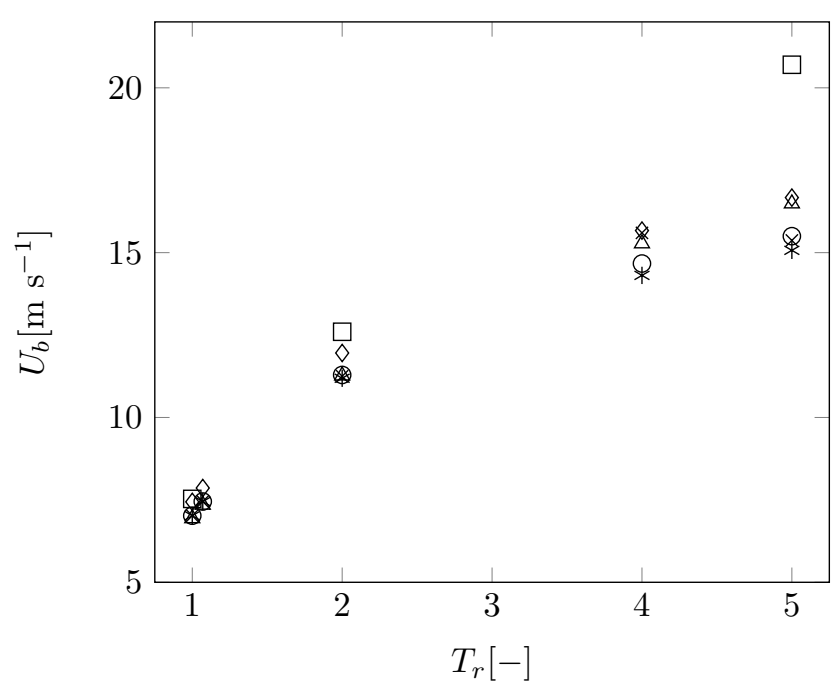

(b) $\operatorname{Re}_{\tau m}=395$

Figure 5 - Evolution of the bulk velocity as a function of the temperature ratio $T_{r}$ 


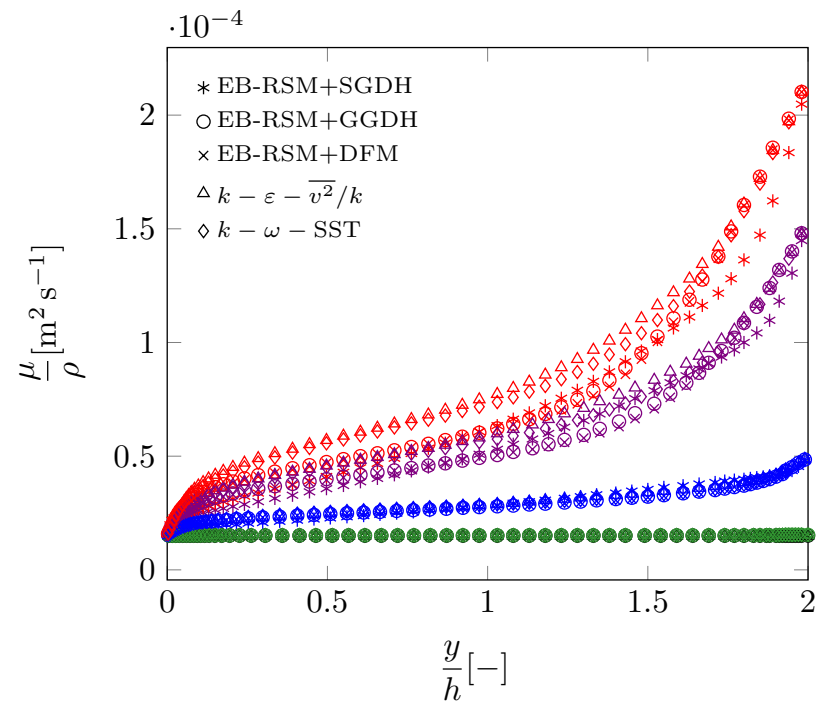

(a) $\operatorname{Re}_{\tau m}=180$

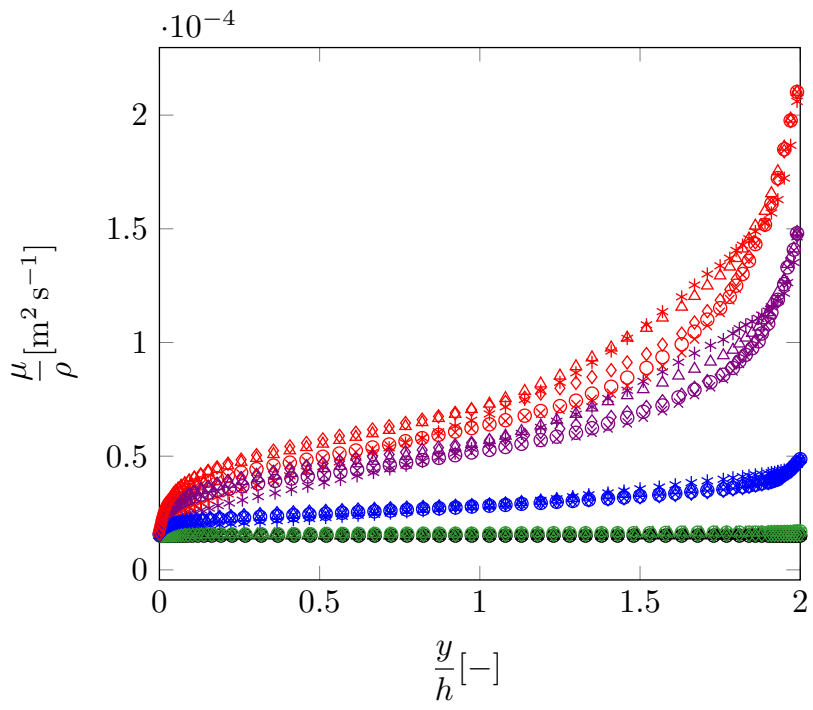

(b) $\operatorname{Re}_{\tau m}=395$

Figure 6 - Evolution of the ratio of the molecular viscosity with the density between the two plates at the two friction Reynolds numbers $\operatorname{Re}_{\tau m}=180$ (left) and 395 (right) for the four temperature ratios $T_{r}=1$ (black), 1.01/1.07 (green), 2 (blue) and 5 (red)

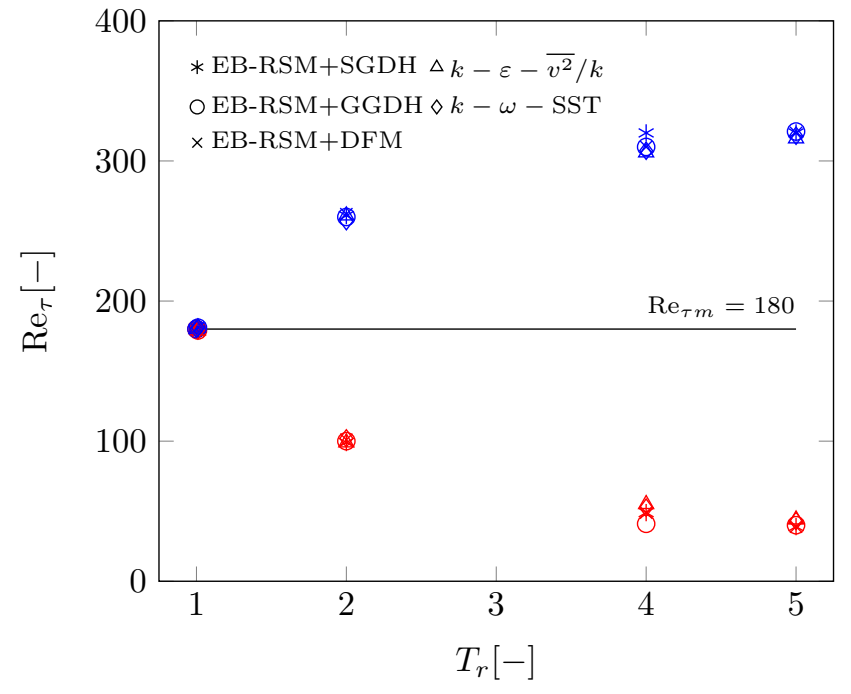

(a) $\operatorname{Re}_{\tau m}=180$

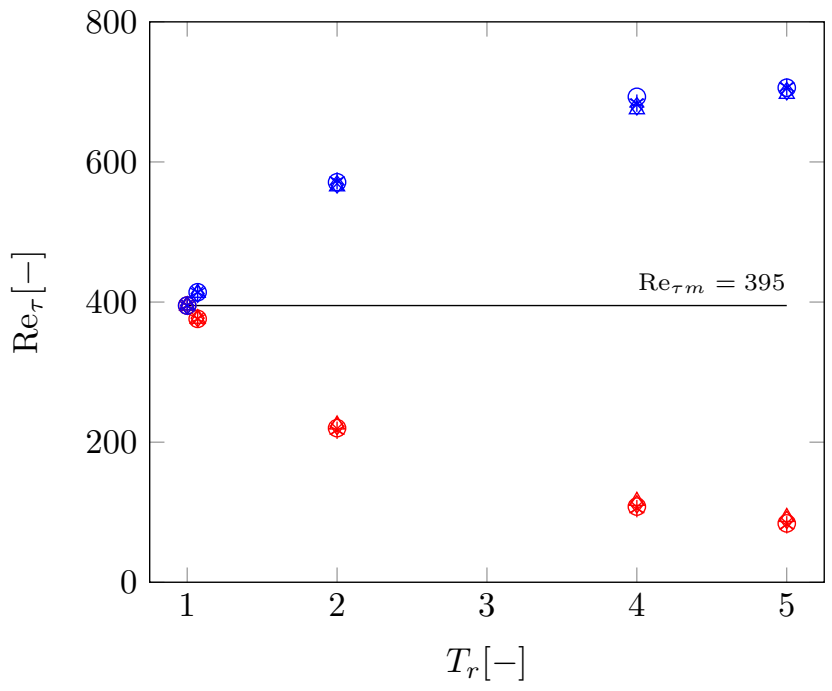

(b) $\operatorname{Re}_{\tau m}=395$

Figure 7 - Evolution of the friction Reynolds numbers for the cold wall (1, blue) and the hot wall (2, red) as a function of the temperature ratio $T_{r}$ 


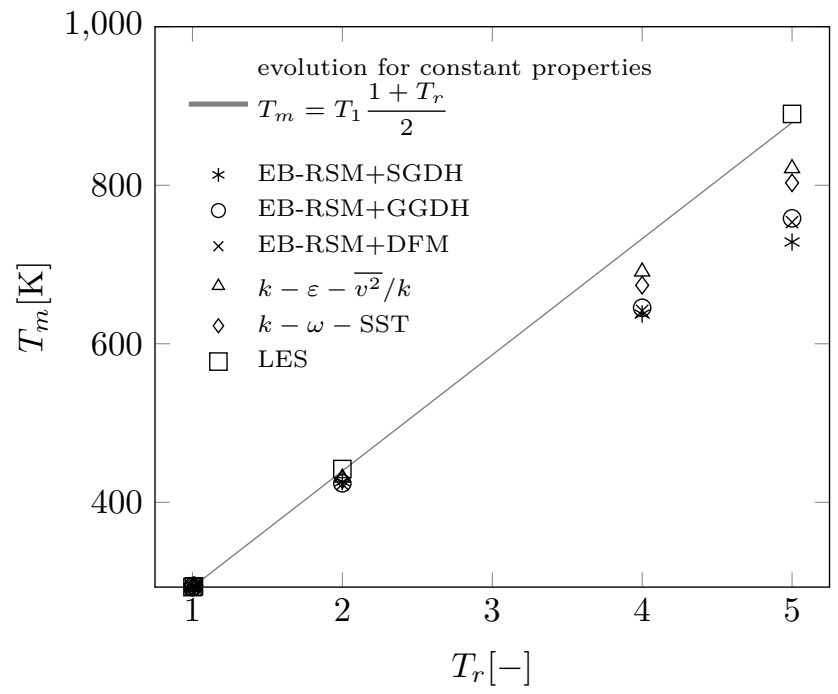

(a) $\operatorname{Re}_{\tau m}=180$

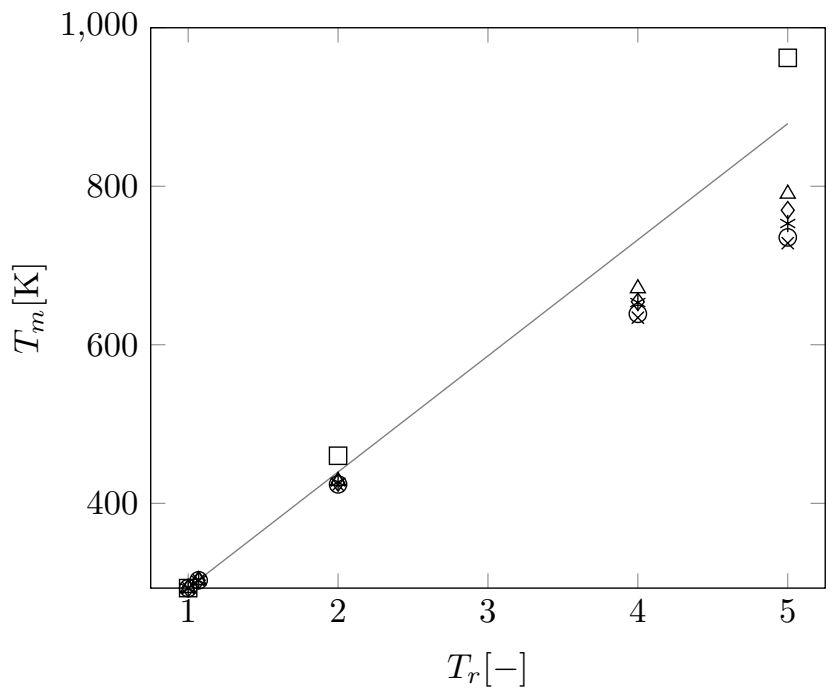

(b) $\operatorname{Re}_{\tau m}=395$

Figure 8 - Evolution of the mean temperature as a function of the temperature ratio $T_{r}$ 


\subsubsection{Velocity field}

Now, the evolution of the mean velocity with transverse temperature gradients is investigated. In order to highlight the main phenomena and also to further understand the underlying mechanisms, a theoretical analysis is first performed for the case of a laminar flow.

Thereby, a fully developed Poiseuille flow is considered, with a transverse temperature gradient. This problem obeys the Poisson equation:

$$
\frac{\partial}{\partial x_{j}}\left(\mu(T) \frac{\partial U_{i}}{\partial x_{j}}\right)=\frac{\partial P}{\partial x_{i}} .
$$

For the fully developed case, the flow is one-directional and oriented along the $x$-axis, and gradients are in the transverse direction, such that

$$
\frac{d}{d y}\left(\mu(T) \frac{d U}{d y}\right)=\psi
$$

where $y$ stands for the distance to the wall.

By integrating across the channel, one gets

$$
\mu(T) \frac{d U}{d y}=\left(\frac{\tau_{w, 1}}{\tau_{w}}-\frac{y}{h}\right) \tau_{w},
$$

such that the shear stress varies linearly across the channel, as shown in figure 9 In the case of a constant viscosity, integrating equation 4.16 leads to:

$$
U_{2 h}=U_{0}+\frac{1}{\mu} \int_{0}^{2 h}\left(\frac{\tau_{w, 1}}{\tau_{w}}-\frac{y}{h}\right) \tau_{w} d y=\frac{1}{\mu} \int_{0}^{2 h}\left(\frac{\tau_{w, 1}}{\tau_{w}}-\frac{y}{h}\right) \tau_{w} d y .
$$

Since the velocity is zero on the upper wall, the integral must cancel, which means that the surface under the curve (see figure 9(a) vanishes. Consequently, this corresponds to a symmetrical situation, with $\frac{\tau_{w, 1}}{\tau_{w}}=1$, i.e. the surfaces on either side of the zero crossing location compensate for each other.

In the present case where the temperature increases with $y$ and given the Sutherland law 2.3a), the dynamic viscosity also with $y$. Thus, the integration of 4.16 now reads

$$
U_{2 h}=\int_{0}^{2 h} \frac{1}{\mu(y)}\left(\frac{\tau_{w, 1}}{\tau_{w}}-\frac{y}{h}\right) \tau_{w} d y
$$

Due to the variable viscosity in the integrand, the weight of the left part of the surface will be increased compared to the weight of the right part. Therefore, to guarantee that $U_{2 h}=0, \frac{\tau_{w, 1}}{\tau_{w}}$ must be less than unity in order to reduce the left surface and increase the right surface, as can be seen in figure 9(b)] This implies that, for a laminar flow, the location of the maximal velocity, which corresponds to the point where the shear stress is zero, is shifted towards the left, that is to say towards the cold wall. Figure 10 illustrates this shifting of the velocity profile for increasing temperature ratios. 


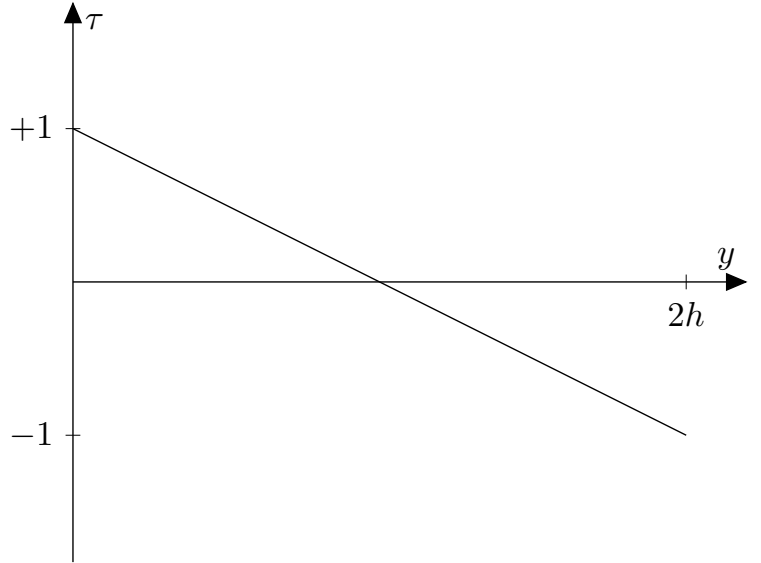

(a) Isothermal case.

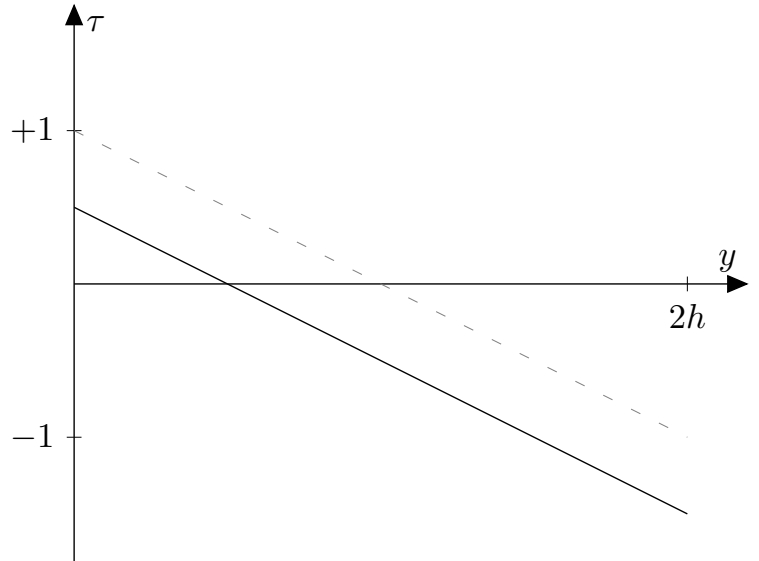

(b) Non-isothermal case.

Figure 9 - Evolution of the shear stress along the transverse direction in the laminar case

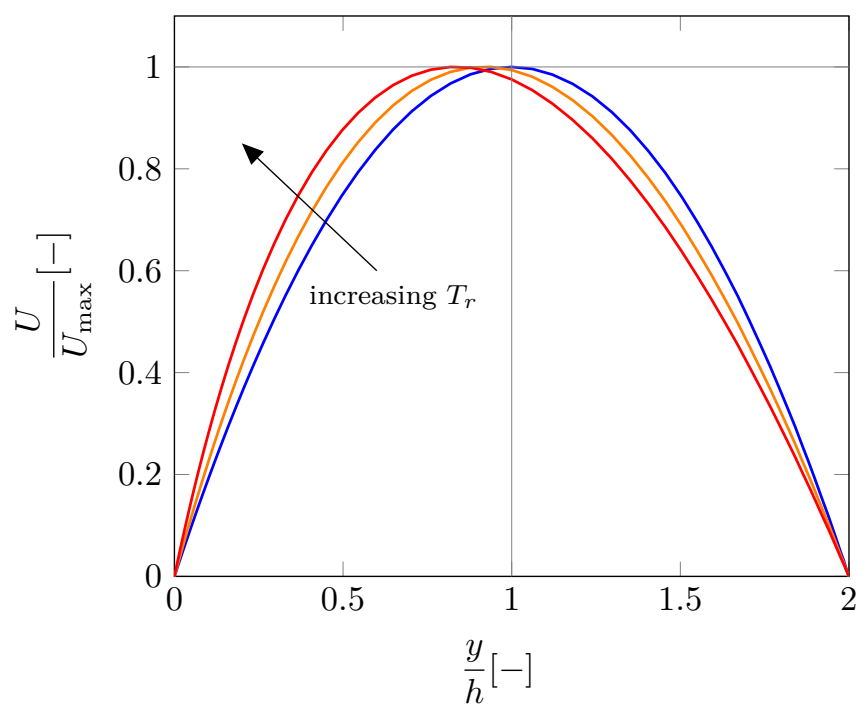

Figure 10 - Influence of the temperature gradient on the longitudinal velocity in laminar flows 
In figure 11, the longitudinal velocity is depicted for all the friction Reynolds numbers and temperature ratios. The RANS results fairly represent the DNS data up to a temperature ratio $T_{r}=2$. For the highest temperature ratio, i.e. $T_{r}=5$, one can see that discrepancies occur between the RANS and the LES results. However, as mentioned above, LES are not sufficiently accurate and are only shown here for qualitative comparison, since no DNS data are available. Furthermore, it can be noted that all the RANS models give very similar results except for the case Re $\cos _{\tau m}=395$ and $T_{r}=5$.

Besides, it is worth mentioning here that the maximum shift is now on the hot side, which means that the behavior is completely different from the laminar regime. Once again, this is in accordance with already existing conclusions [28, 29]. Finally, the asymmetry of the velocity profile is more pronounced for higher temperature ratios, specifically for the lower friction Reynolds number.

To explain the different behavior observed in laminar and turbulent flows, the dynamic viscosity and density are plotted along the transverse axis (i.e. from the cold to the hot wall) in figures 12 and 13 . It can be observed that the molecular viscosity varies between fixed values at the walls, directly linked to the temperature ratio, due to the the Sutherland law 2.3a which only depends on temperature. From the cold to the hot side, the value varies significantly, up to a factor of about 2.75. However, one observes discrepant profiles away from the walls, specifically for the highest temperature ratio. Implicitly, this also means that the temperature profiles present some differences for the various RANS models. Since the averaged thermodynamic pressure $\overline{P_{0}}$ is a constant in the present case, density is directly proportional to the reciprocal of the temperature and reaches values at the wall independent of the RANS model, as observed in Figure 13 . The combined increase of the viscosity and decrease of the density lead to strong variations of the ratio $\mu / \rho$, as mentioned in section 4.2 .1

Moreover, the molecular viscosity is smaller than the turbulent viscosity by one or two orders of magnitude, depending on the Reynolds number, such that its influence is limited to very narrow regions close to the walls. Then, the turbulent viscosity shows a behavior very different from the molecular viscosity. This can be seen in figure 12 , where the turbulent viscosity is plotted for all the models. For the EB-RSM, the eddy-viscosity concept is not used to model the Reynolds stress. For comparison with eddy-viscosity models, the plotted quantity is $\left(\rho \overline{U_{1}^{\prime} U_{2}^{\prime}}\right) / \frac{\mathrm{d} \overline{U_{1}}}{\mathrm{~d} y}$. This quantity can go to infinity if the velocity gradient and the turbulent shear-stress do not vanish at the same location, which occurs when the profiles become asymmetric. Then, one can see that it is strongly reduced near the hot wall, while the molecular viscosity is increased. It can be seen in figure 12 (c) to 12 (e) that, the higher the temperature ratio and the smaller the turbulent Reynolds number, the larger the gap between the cold and hot sides. In line with the previous discussion for the laminar regime, the velocity profile is determined by:

$$
\bar{U}_{2 h}=\int_{0}^{2 h} \frac{1}{\mu+\mu_{t}}\left(\frac{\tau_{w, 1}}{\tau_{w}}-\frac{y}{h}\right) \tau_{w} d y
$$

Yet, in the turbulent regime, the turbulent viscosity dominates the flow behavior and therefore, given the previous remark, the asymmetry of the turbulent viscosity leads to a shift of the maximum of velocity towards the hot side, as can be seen in figure 11, in contrast with the laminar case. Eventually, the turbulent viscosity being significantly reduced near the hot wall, the extent of the region where the molecular viscosity is not negligible increases with the temperature ratio. Thus, this could explain the possible relaminarization of the flow mentioned near the hot wall [28, 29]. For the sake of completeness, the profiles for the turbulent kinetic energy and the dissipation rate are furnished in figures 18 to 19 in the appendix.

The last characteristic to be analyzed is the non-dimensional longitudinal velocity $U^{+}$, which is depicted in figure 14. For moderate temperature ratios, see figure 14(a), there are virtually no difference between the cold and hot sides (therefore only one side is exploited) and the results agree only for the smaller Reynolds number case. Then, only the $k-\varepsilon-\overline{v^{2}} / k$ model underestimates the velocity in the log layer. When the thermal gradient becomes strong, i.e. when $T_{r} \geq 2$ (see figures $14(\mathrm{~b})$ and 14(c) , then the flow behavior is different at the cold and the hot side, correlatively to what was seen before for the friction Reynolds number in figure 7 Besides, it is even more interesting to note that profiles do not follow anymore the standard (isothermal) law-of-the-wall, as pointed out by other studies [1,24, 28, 55, 58, on the cold side, the slope of the velocity profile is higher, whereas for the hot side it is lower, in particular for the lower friction Reynolds number and the higher temperature ratio. Furthermore, the comparison with the available DNS results is satisfactory for all models, contrary to the LES for the cold side. Moreover, for the lower friction Reynolds number, all RANS models are in close agreement except for the $k-\varepsilon-\overline{v^{2}} / k$ on the cold side for the higher temperature ratio. For the higher friction Reynolds number, some discrepancies appear between the Reynolds-stress and eddy-viscosity models. Unfortunately, in the absence of reliable reference data, no general conclusion can be drawn concerning the relative performance of the models. 

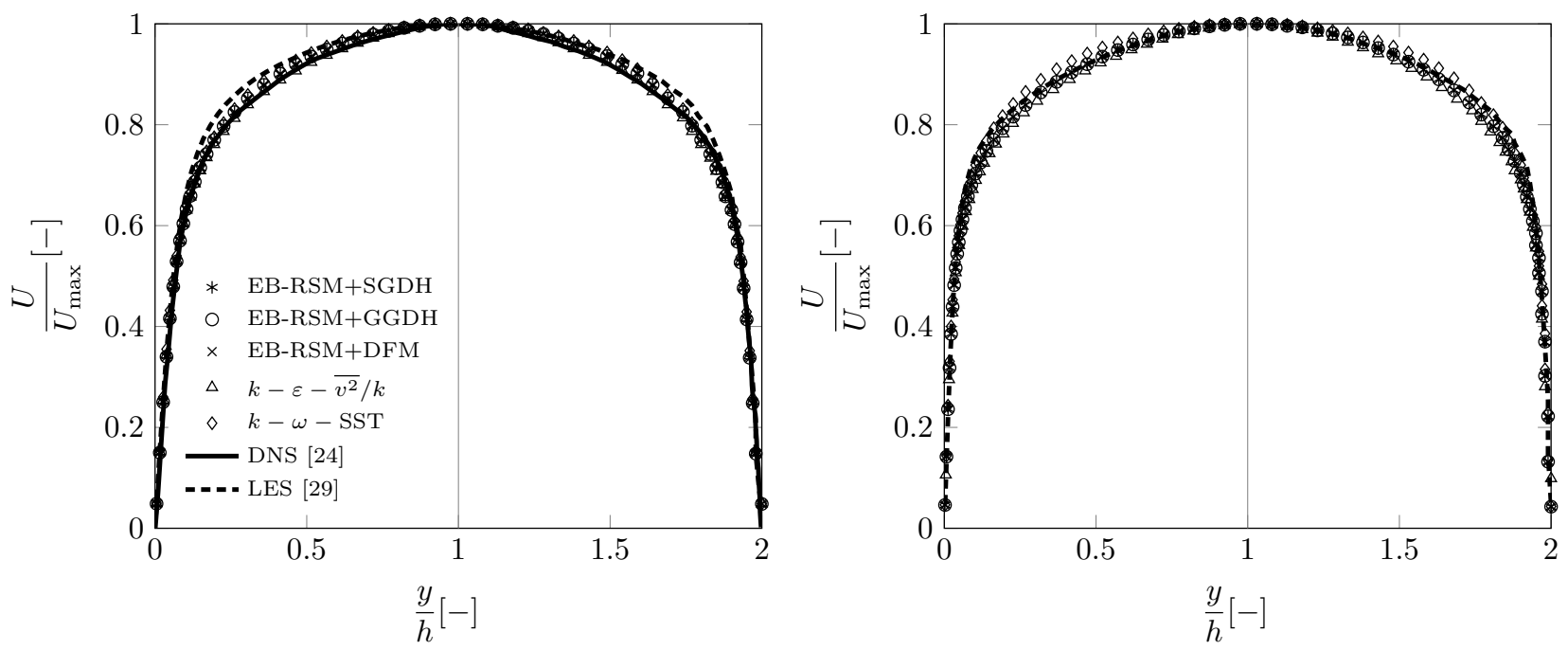

(a) $\operatorname{Re}_{\tau m}=180 \& T_{r}=1.01$ (left) and $\operatorname{Re}_{\tau m}=395 \& T_{r}=1.07$ (right)
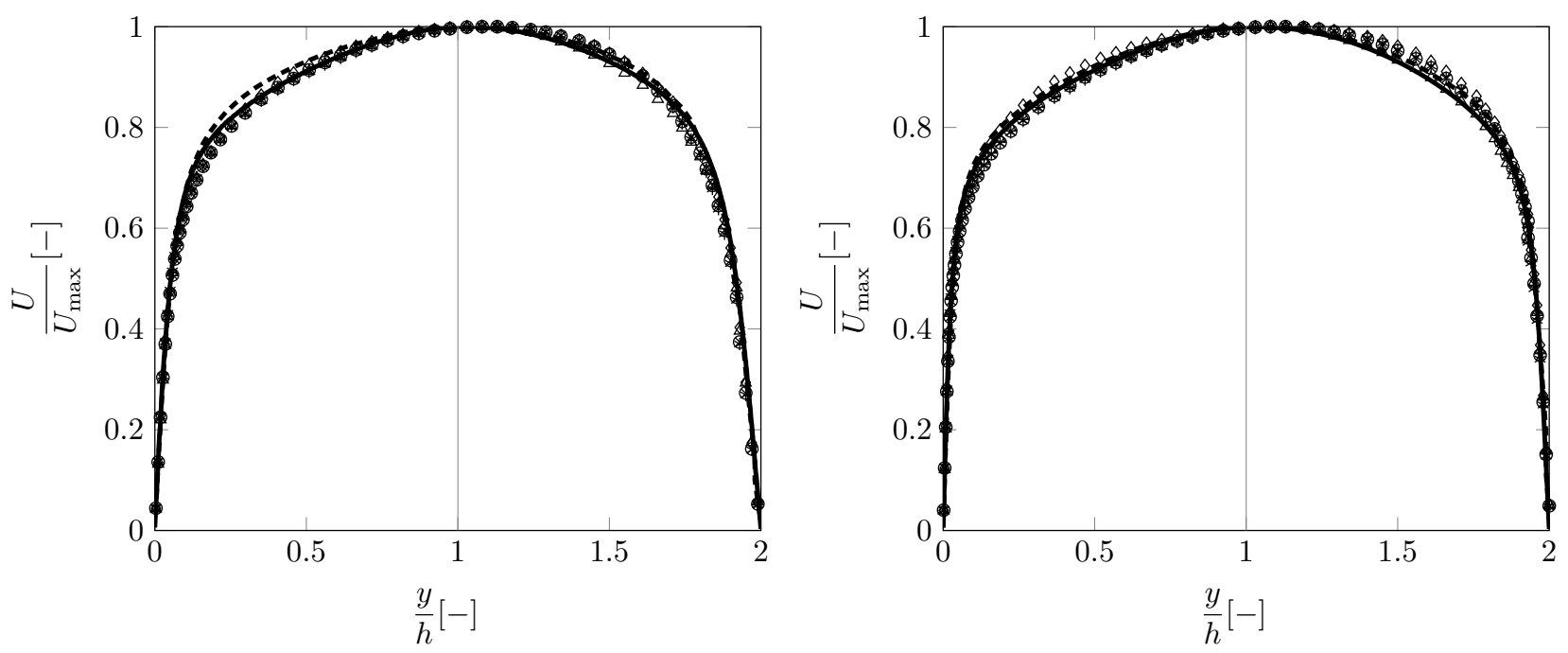

(b) $\operatorname{Re}_{\tau m}=180$ (left) and 395 (right) for $T_{r}=2$
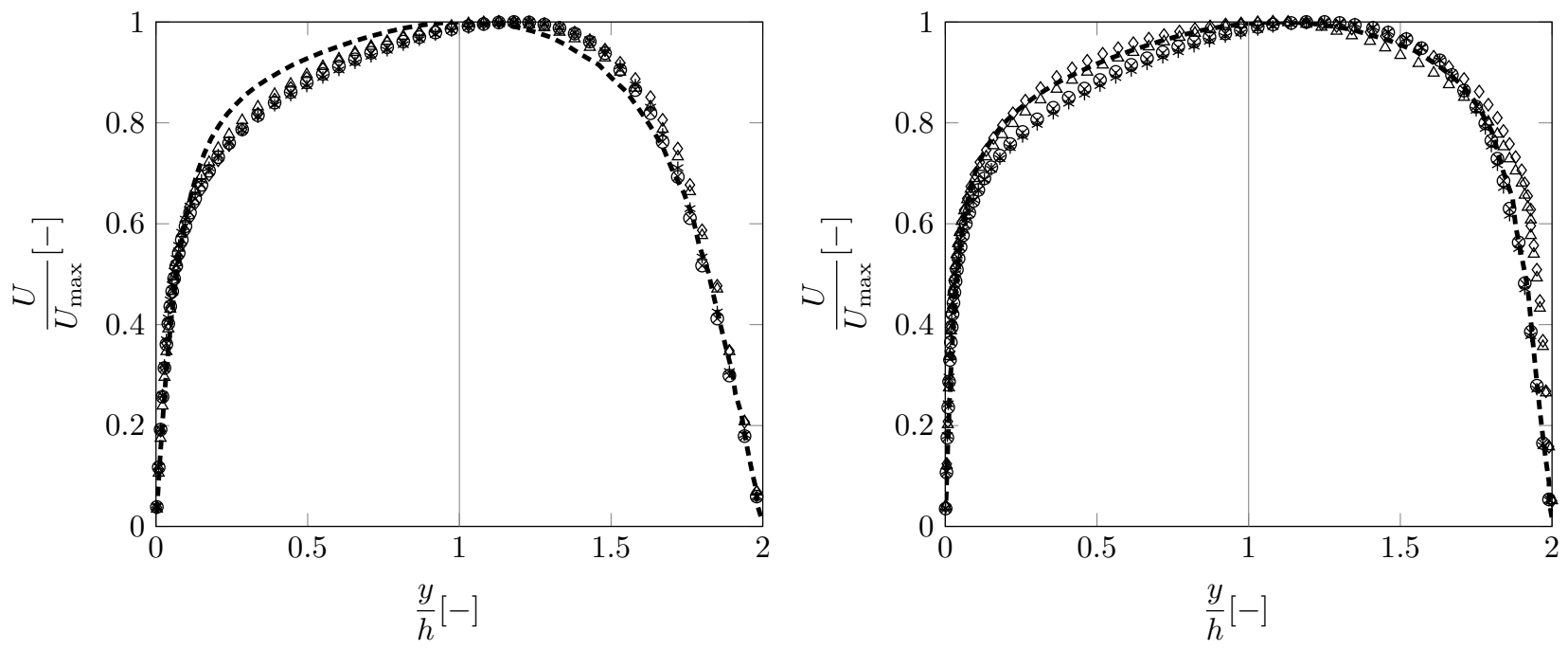

(c) $\operatorname{Re}_{\tau m}=180$ (left) and 395 (right) for $T_{r}=5$

Figure 11 - Non-dimensional longitudinal velocity $U / U_{\max }$ along the non-dimensional transverse direction $y / h$ 

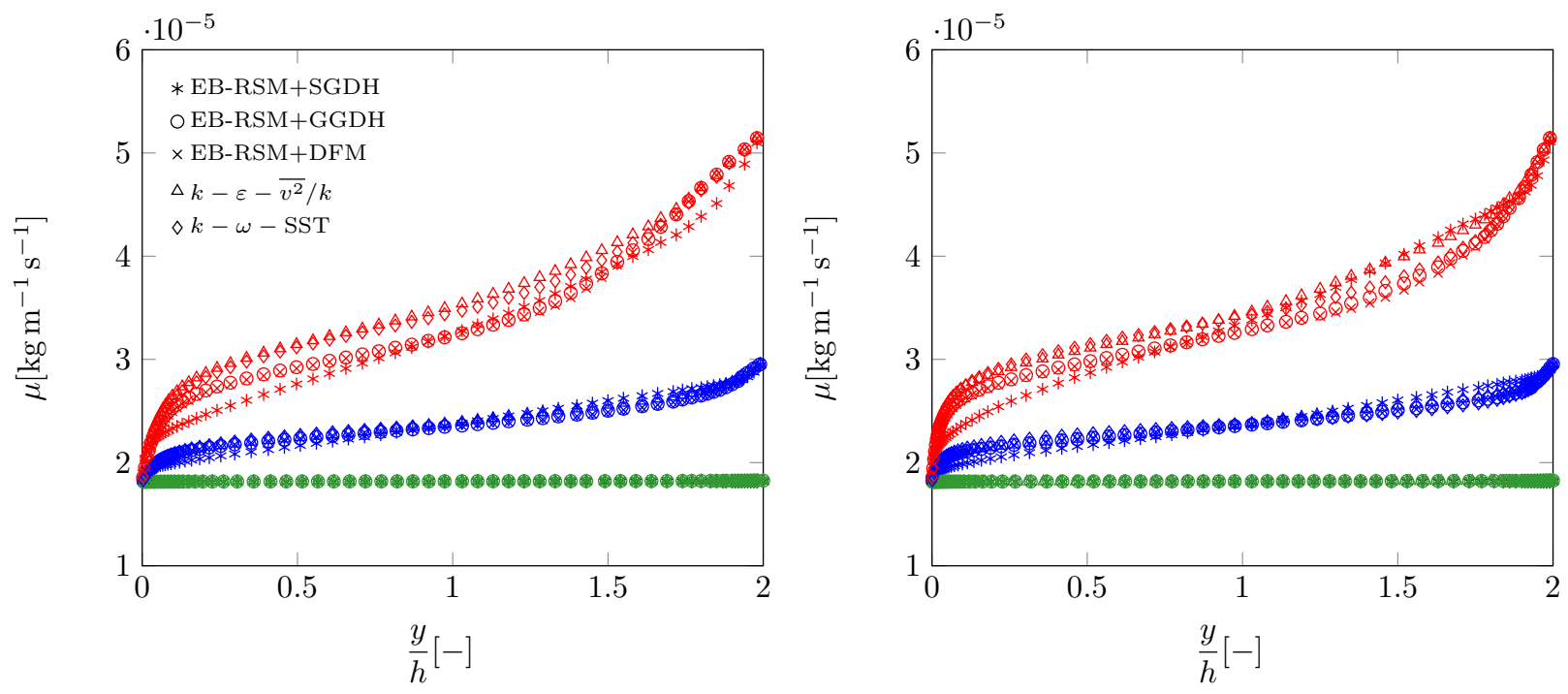

(a) Molecular viscosity
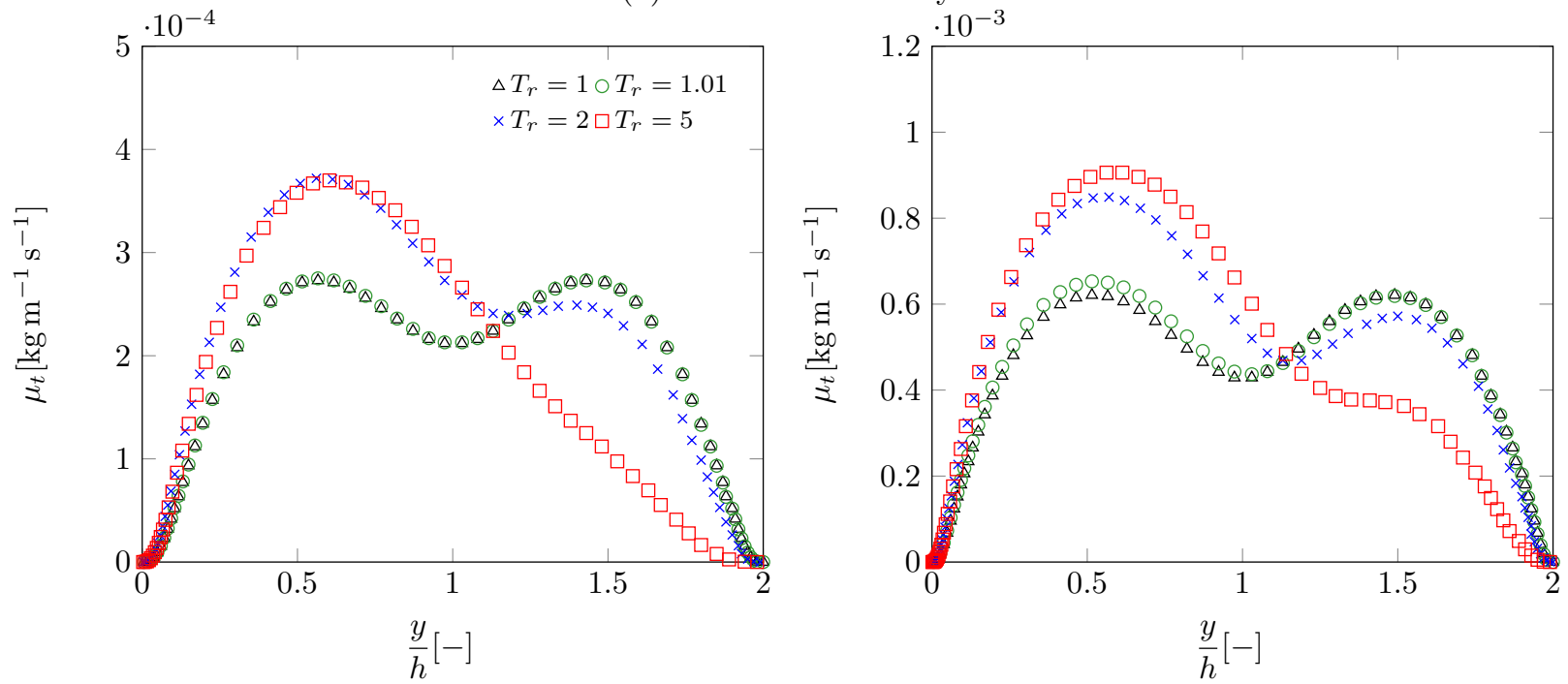

(b) Turbulent viscosity for $k-\varepsilon-\overline{v^{2}} / k$
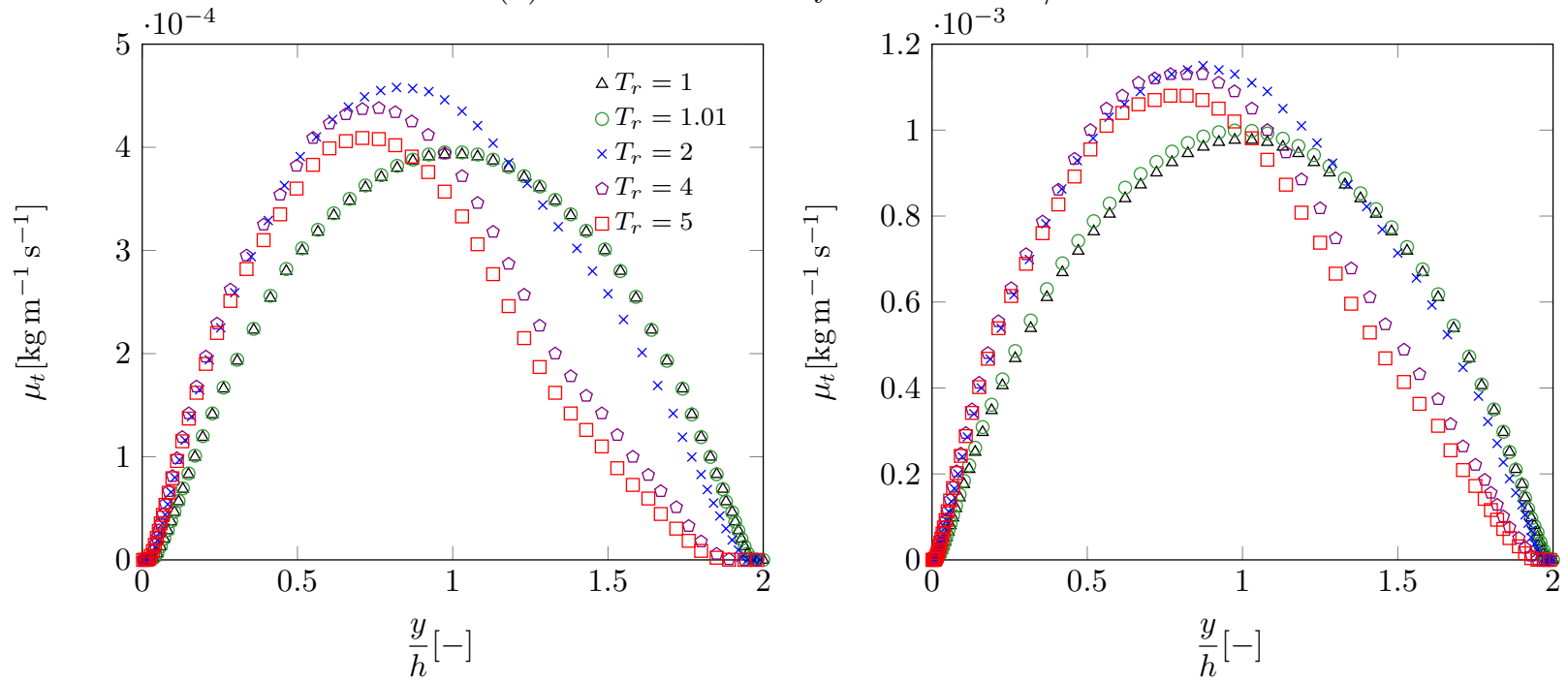

(c) Turbulent viscosity for $k-\omega-\mathrm{SST}$

Figure 12: see below... 

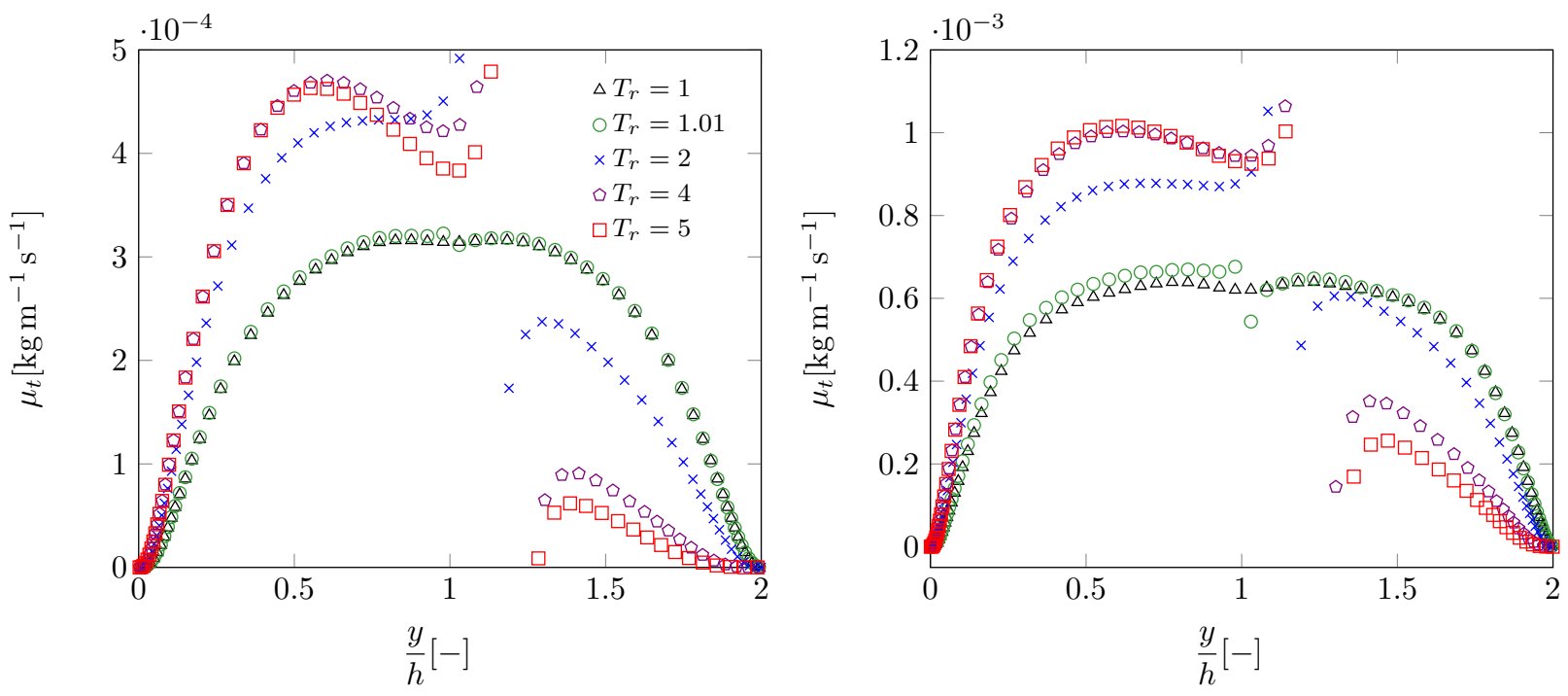

(d) Turbulent viscosity for EB-RSM+SGDH
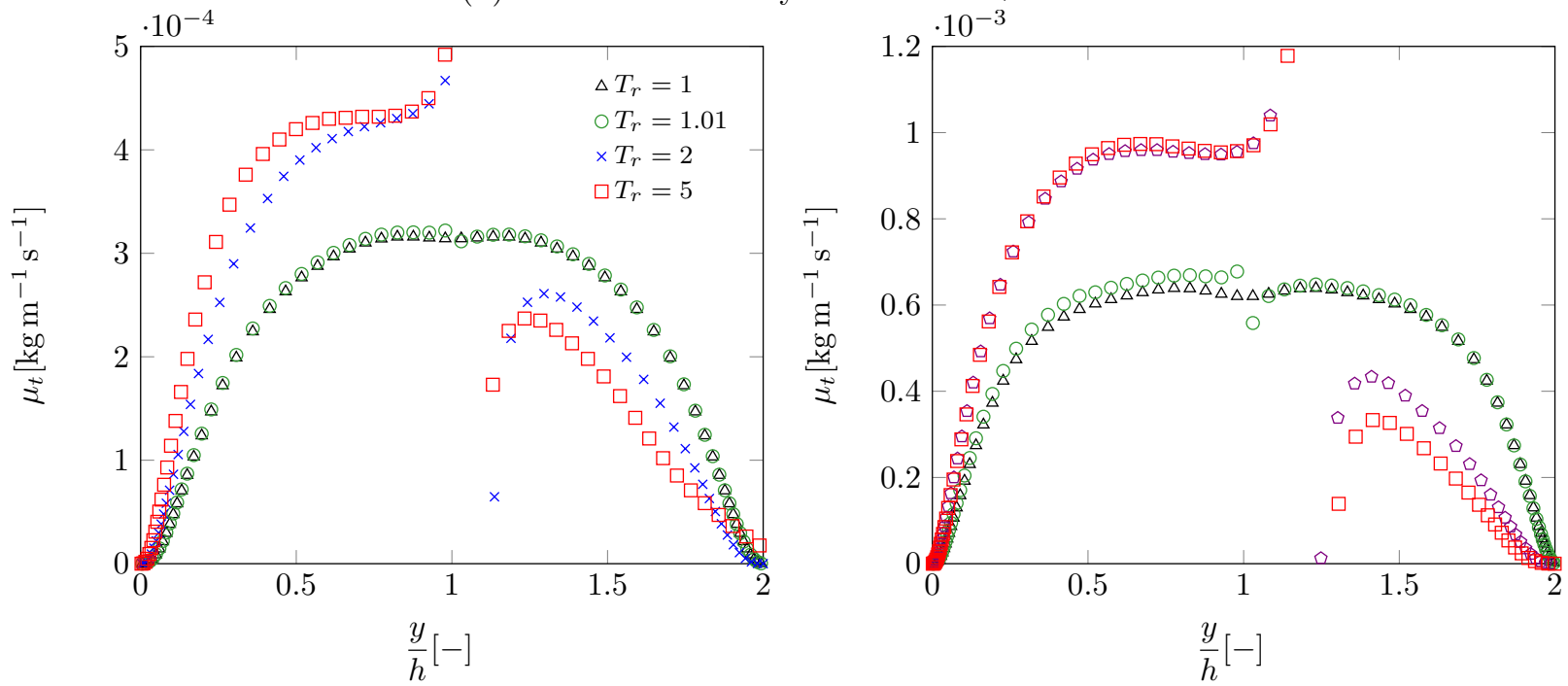

(e) Turbulent viscosity for EB-RSM+GGDH
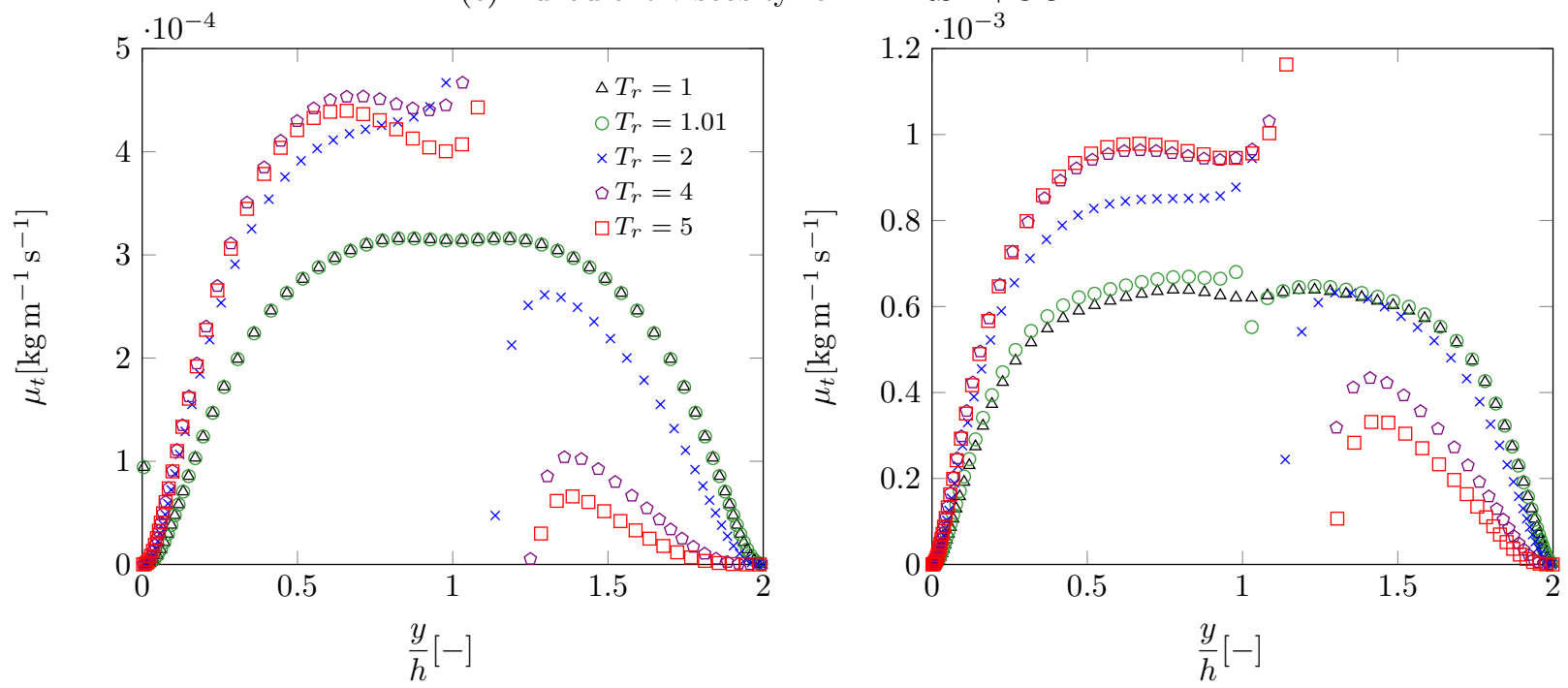

(f) Turbulent viscosity for EB-RSM+DFM

Figure 12: Evolution of the dynamic viscosities between the two plates at the two friction Reynolds numbers $\operatorname{Re}_{\tau m}=180$ (left) and 395 (right) for various temperature ratios $T_{r}$ 

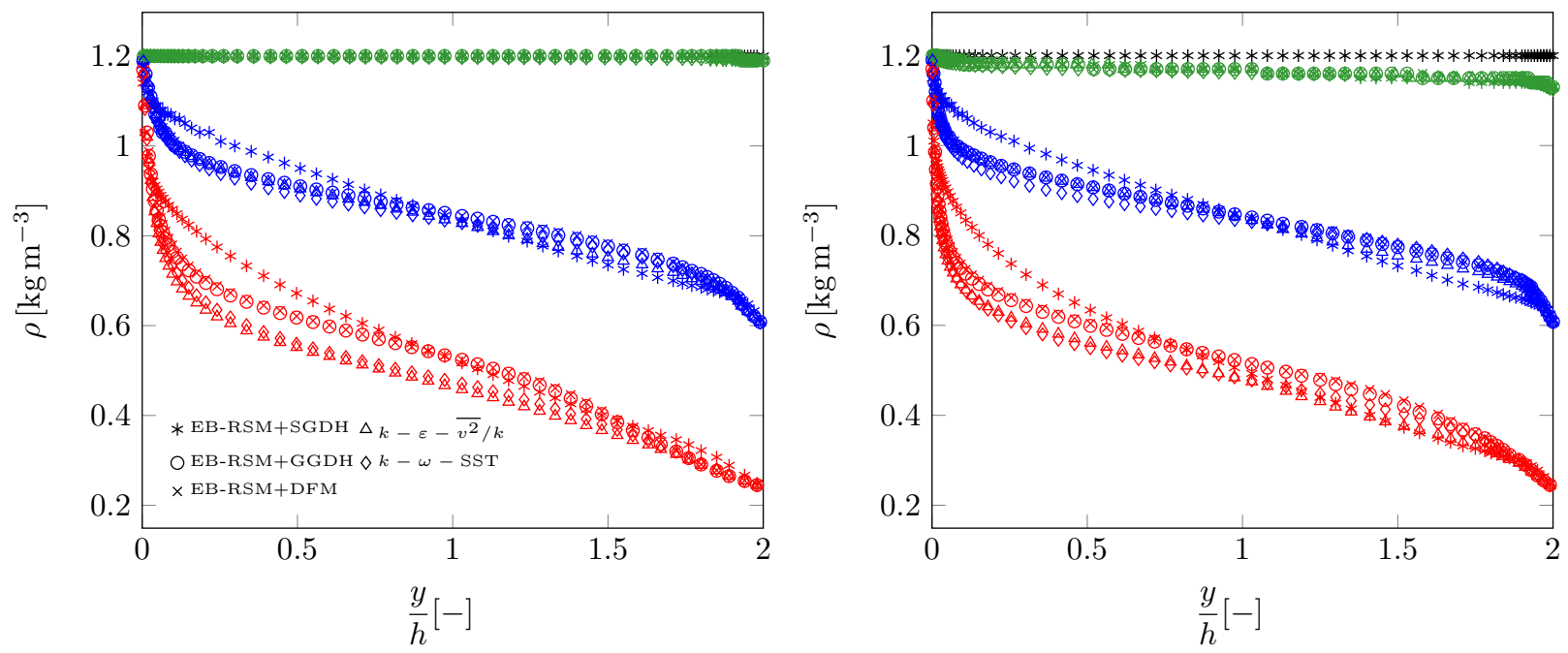

Figure 13 - Evolution of the density between the two plates at the two friction Reynolds numbers $\operatorname{Re}_{\tau m}=180$ (left) and 395 (right) for the four temperature ratios, from top to bottom: $T_{r}=1$ (black), $1.01 \& 1.07$ (green), 2 (blue) and 5 (red) 

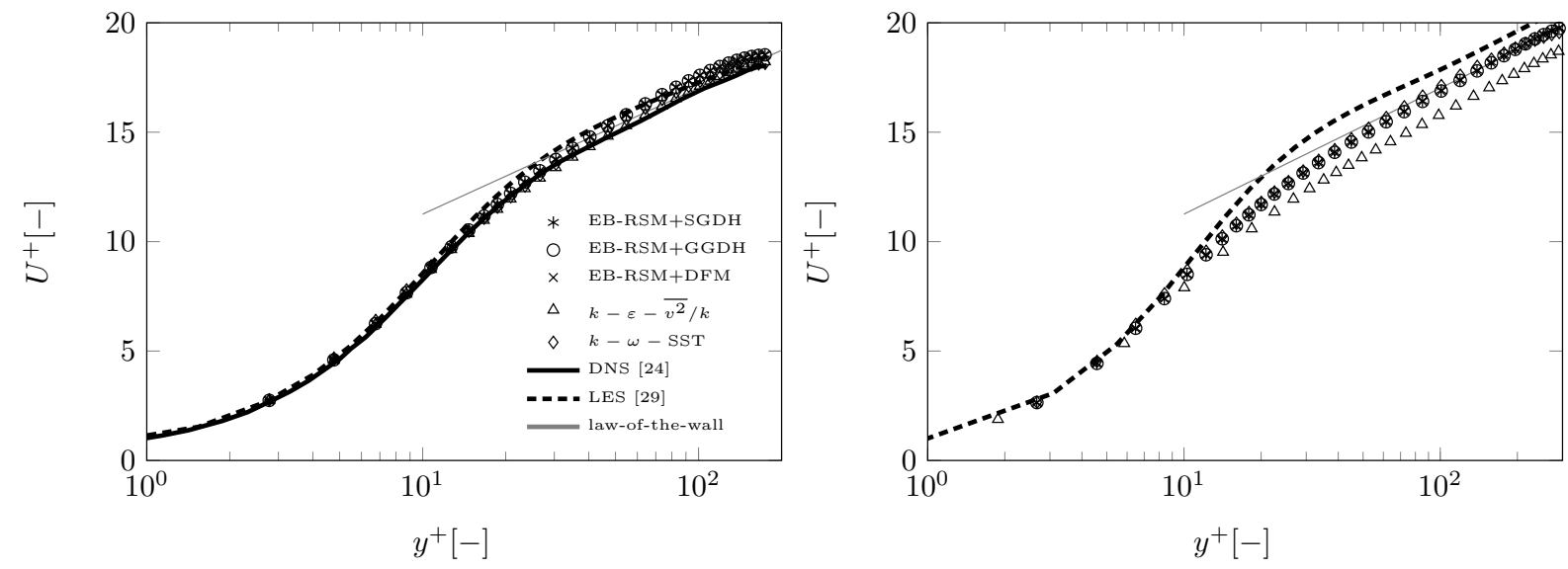

(a) $\operatorname{Re}_{\tau m}=180 \& T_{r}=1.01$ (left) and $\operatorname{Re}_{\tau m}=395 \& T_{r}=1.07$ (right)
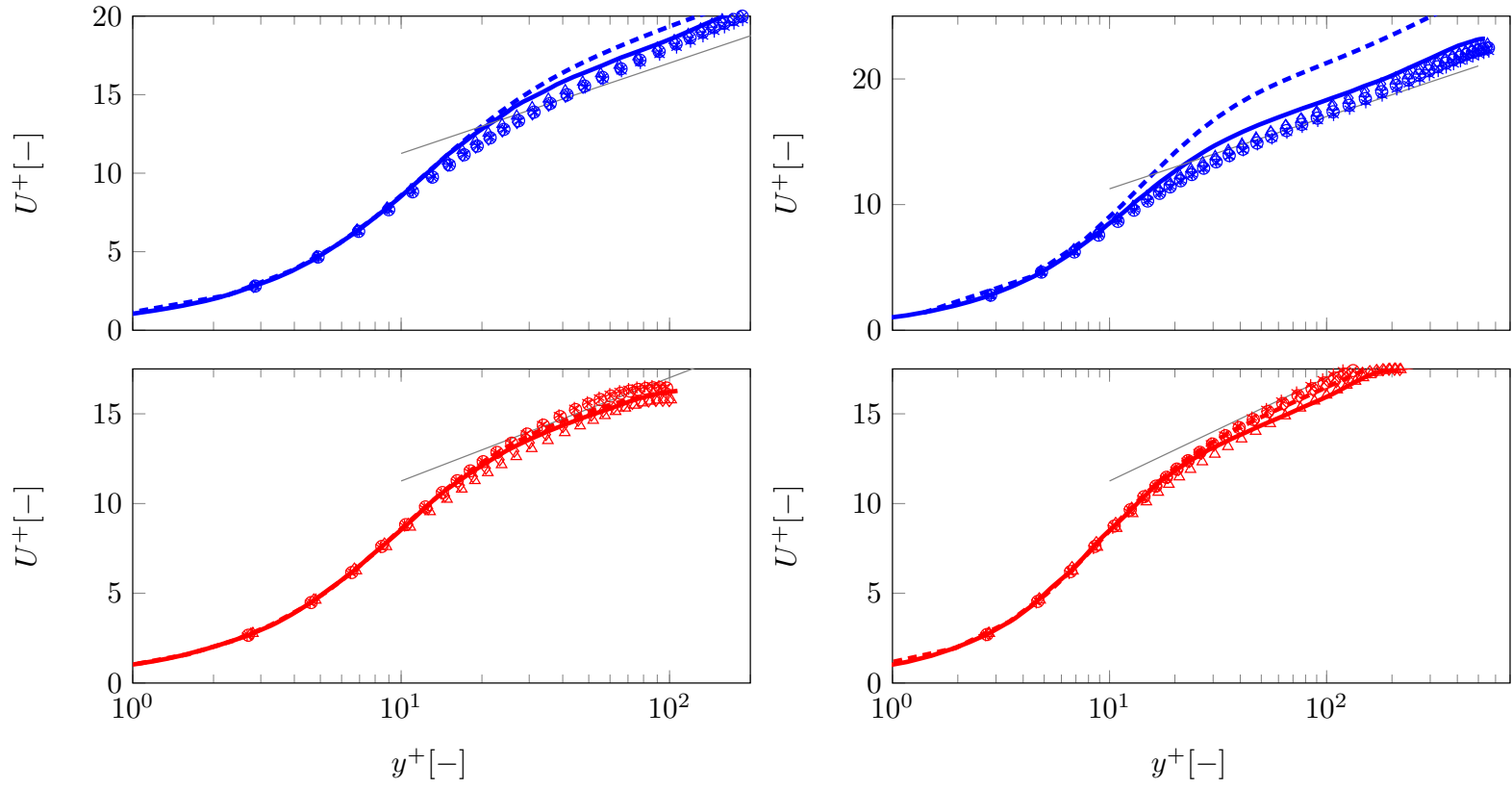

(b) $\operatorname{Re}_{\tau m}=180$ (left) and 395 (right) for $T_{r}=2$
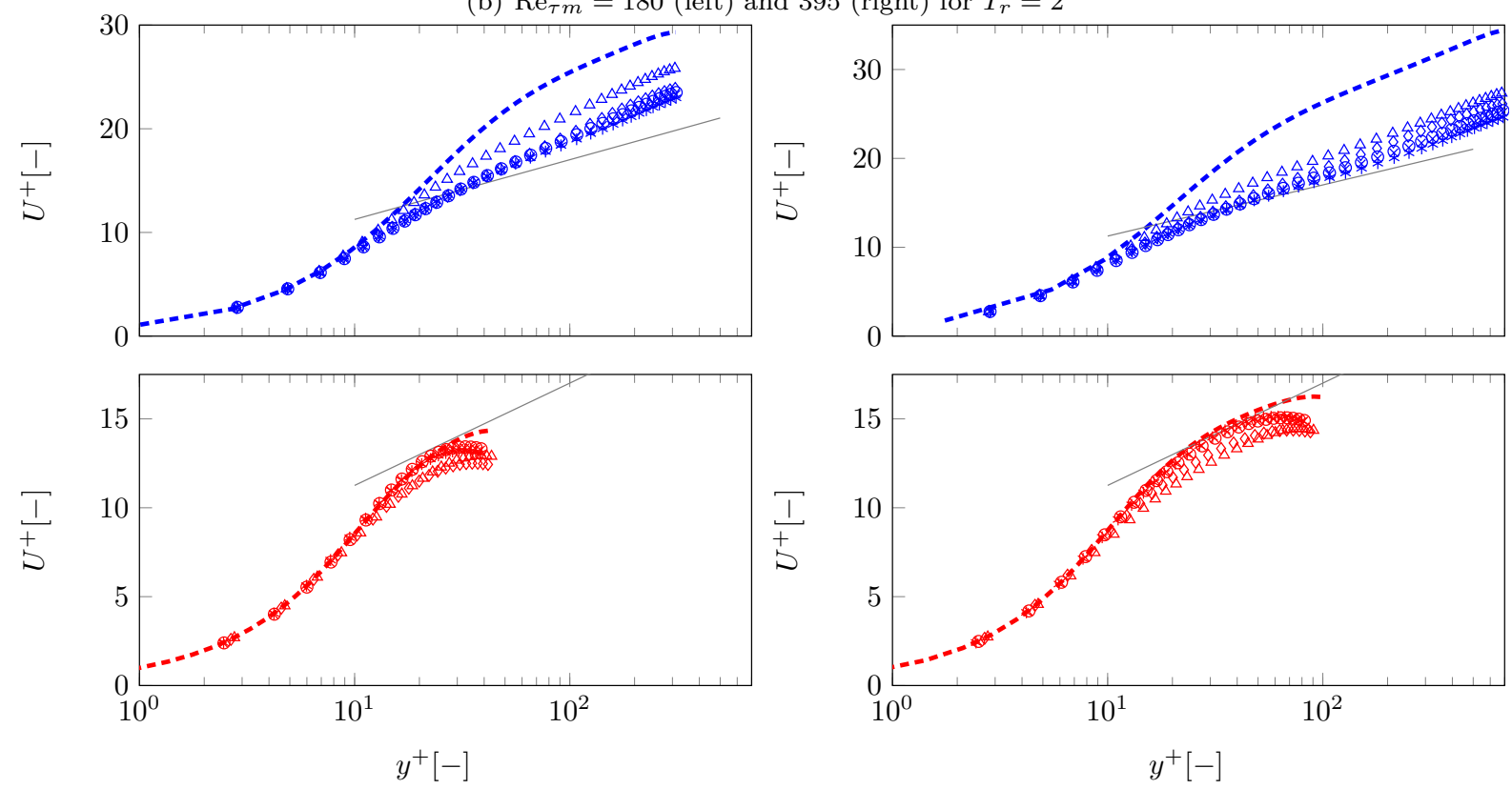

(c) $\operatorname{Re}_{\tau m}=180$ (left) and 395 (right) for $T_{r}=5$

Figure 14 - Non-dimensional longitudinal velocity $U^{+}$on the hot (red) and cold (blue) walls in function of $y^{+}$ 


\subsubsection{Temperature field}

After the study of the velocity, this part is now focused on the temperature profiles obtained with the various RANS models. In figures 15 and 16, the temperature profiles are made non-dimensional either by the temperature difference between the cold and the hot wall or by the friction temperature in order to emphasize the behavior of the fluid at the center or near the walls, respectively.

The first remark to be made concerns the results obtained with the SGDH closure associated with the EB-RSM: it clearly fails to reproduce even the qualitative characteristics of the flow, as can be seen in both figures. These discrepancies can go up to $20 \%$ to $40 \%$ compared with the other models. Indeed, since it is a second-moment closure, the EB-RSM does not make use of any eddy viscosity. However, such a parameter has been shown to be needed in the formulation (2.6) for the SGDH, so an eddy viscosity has to be defined and computed. The scalar turbulent diffusivity involved in the model indeed writes:

$$
\alpha_{t}=\frac{\nu_{t}}{\operatorname{Pr}_{t}}
$$

where, for the EB-RSM, $\nu_{t}$ is evaluated as:

$$
\nu_{t}=\frac{1}{4} \frac{{\overline{U_{i}^{\prime} U_{i}^{\prime}}}_{\varepsilon}}{\varepsilon} .
$$

As initially mentioned by [52], this relation is not correct in the near-wall region. This is the main motivation for the development of the $\overline{v^{2}}-f$ family of models 44 in which $\overline{v^{2}}$, the characteristic scale of the energy of the wall-normal turbulent fluctuations, is introduced to correct the expression for the turbulent heat flux.

The corresponding behavior for the turbulent viscosity is thus depicted in figure 17. Due to the use of this incorrect relation, the SGDH leads to erroneous results for the thermal field when used with the EB-RSM. In contrast, if one now looks at the $k-\varepsilon-\overline{v^{2}} / k$ model, it appears that relation 2.10a can be injected into formulation 2.6. that can be written as

$$
{\overline{U_{i}^{\prime} T^{\prime}}}_{k-\varepsilon-\overline{v^{2}} / k-\mathrm{SGDH}}=\frac{C_{\mu}}{\operatorname{Pr}_{t}} \varphi k \min \left(\mathcal{T} ; \mathcal{T}_{\text {lim }}\right) \frac{\partial \bar{T}}{\partial x_{i}} .
$$

In the present case, the stagnation correction is not active, such that

$$
{\overline{U_{i}^{\prime} T^{\prime}}}_{k-\varepsilon-\overline{v^{2}} / k-\mathrm{SGDH}}=\frac{C_{\mu}}{\operatorname{Pr}_{t}} \varphi k \sqrt{\frac{k^{2}}{\varepsilon^{2}}+C_{T}^{2} \frac{\nu}{\varepsilon}} \frac{\partial \bar{T}}{\partial x_{i}} .
$$

Except in the viscous sublayer, the second term under the square-root is low compared to the first one, so one gets:

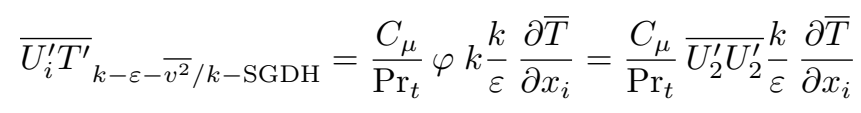

Consequently, it appears that, due to the use of a specific scale in the $k-\varepsilon-\overline{v^{2}} / k$ model, the SGDH expression is similar to the GGDH one defined by relation (2.7). This implies that the choice for an eddy diffusivity relation for second-moment closures, which intrinsically do not need it, is of great importance when performing thermal simulations, and the SGDH must be excluded.

Secondly, it can be seen that, for weak temperature ratios, models very well reproduce the quasi-symmetric temperature profiles except for the LES as can be seen in figure 16(a). Moreover, it is observed in figure 15(b) that LES predicts an increase of the temperature at the center of the channel, whereas all RANS models predict a decrease, in accordance with DNS. Besides, one can note a clear difference between the first-moment closures, on the one hand, and the second-moment closures, on the other hand. When comparing with the available DNS results, a good agreement is obtained on the hot side, yet it seems that first-moment closures are slightly better on the cold side. Finally, discrepancies between RANS models become more pronounced for the highest temperature ratio, specifically on the cold side, as can been observed in figures $15(\mathrm{c})$ and $16(\mathrm{c})$. 

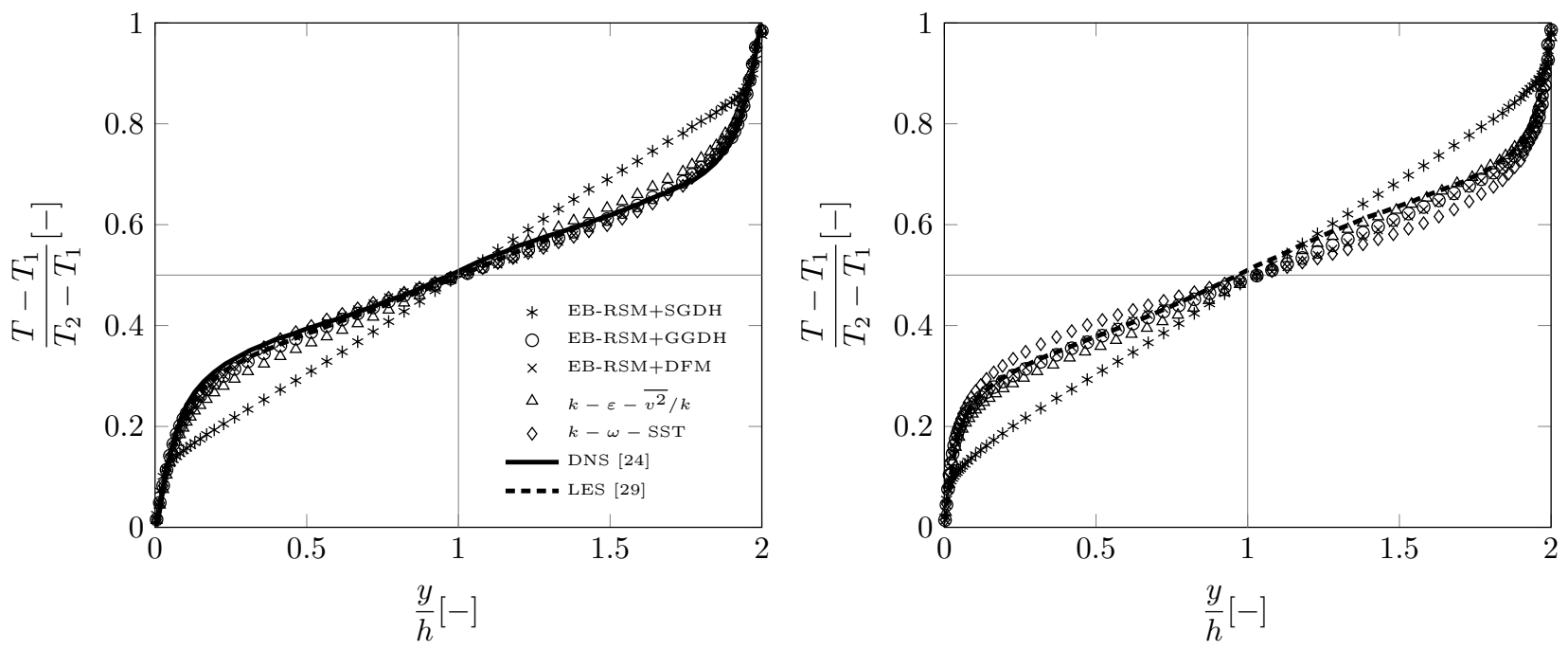

(a) $\operatorname{Re}_{\tau m}=180 \& T_{r}=1.01$ (left) and $\operatorname{Re}_{\tau m}=395 \& T_{r}=1.07$ (right)
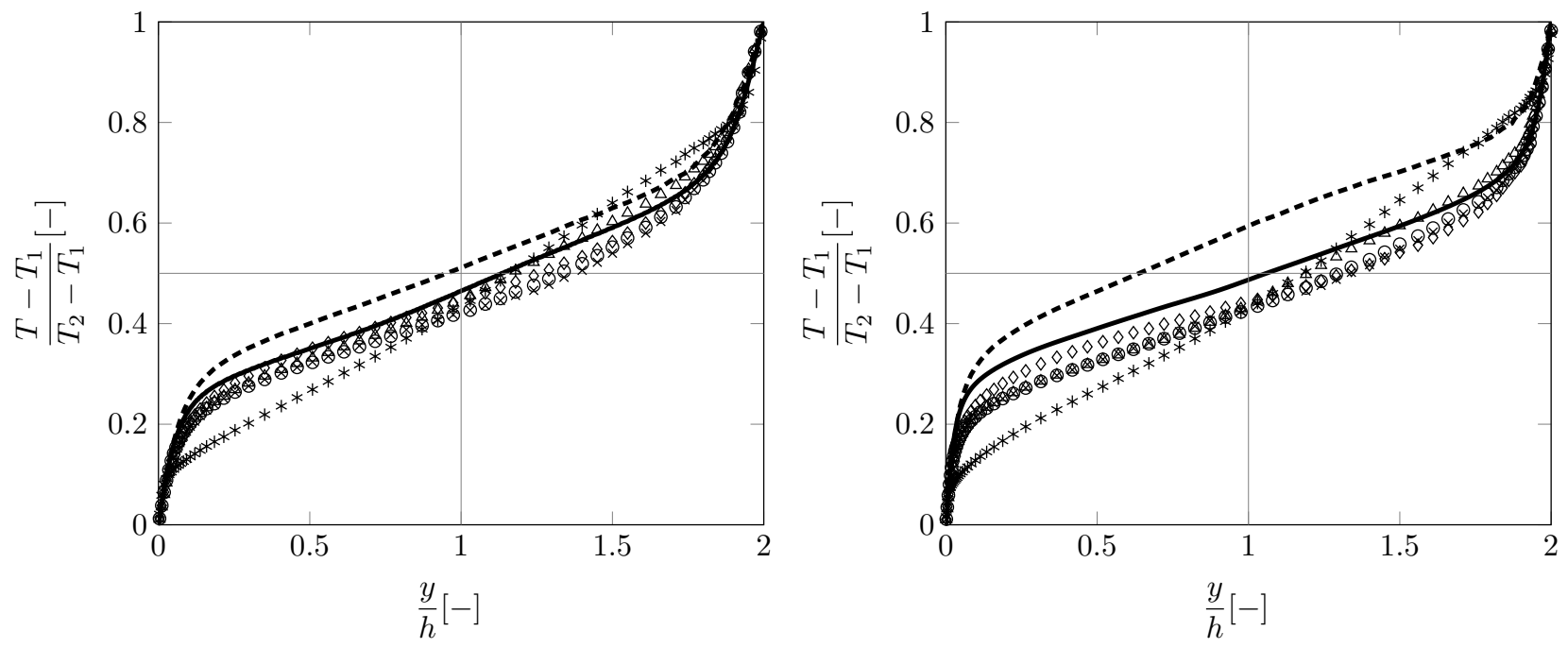

(b) $\operatorname{Re}_{\tau m}=180$ (left) and 395 (right) for $T_{r}=2$
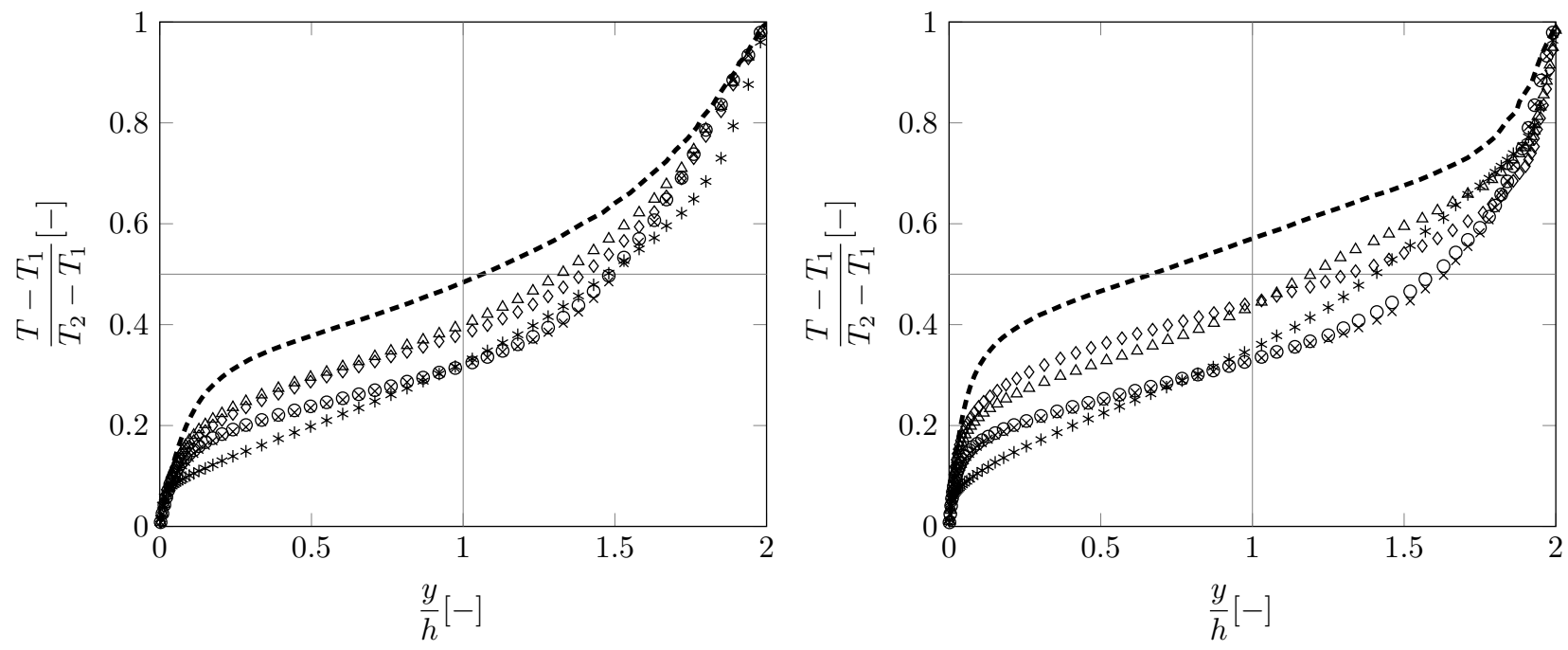

(c) $\operatorname{Re}_{\tau m}=180$ (left) and 395 (right) for $T_{r}=5$

Figure 15 - Non-dimensional temperature $\left(T-T_{1}\right) /\left(T_{2}-T_{1}\right)$ along the non-dimensional transverse direction $y / h$ 

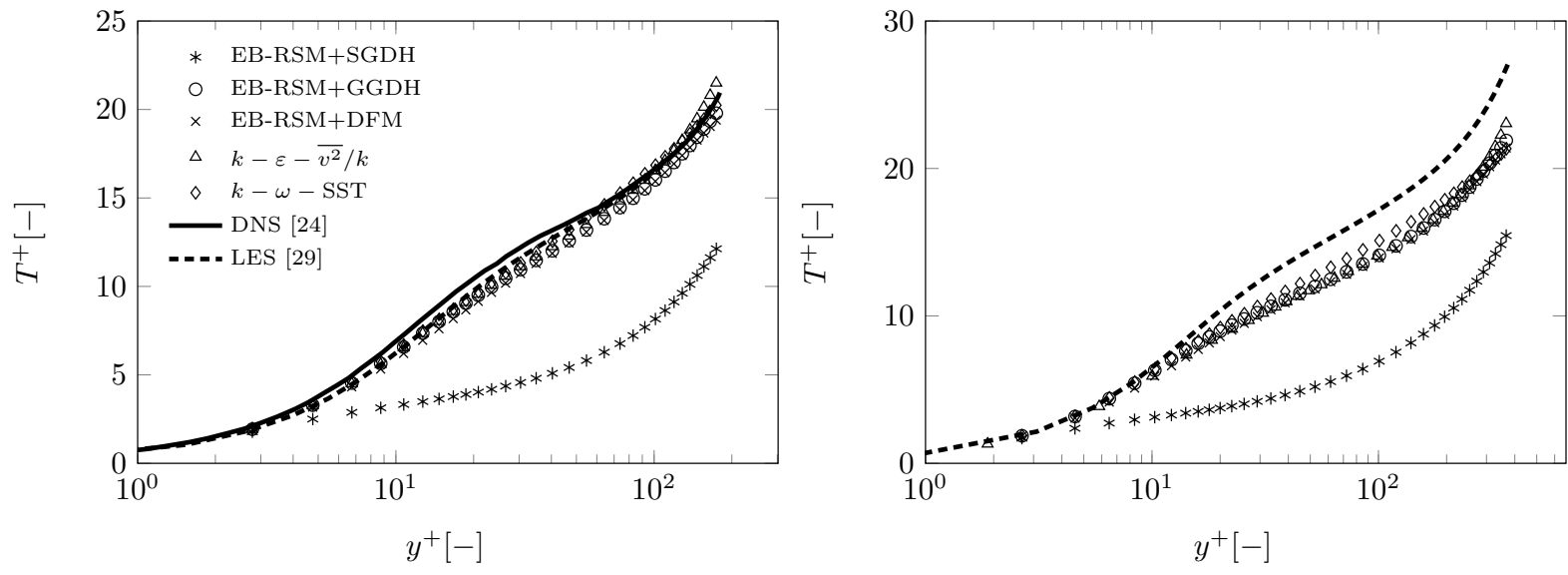

(a) $\operatorname{Re}_{\tau m}=180 \& T_{r}=1.01$ (left) and $\operatorname{Re}_{\tau m}=395 \& T_{r}=1.07$ (right)
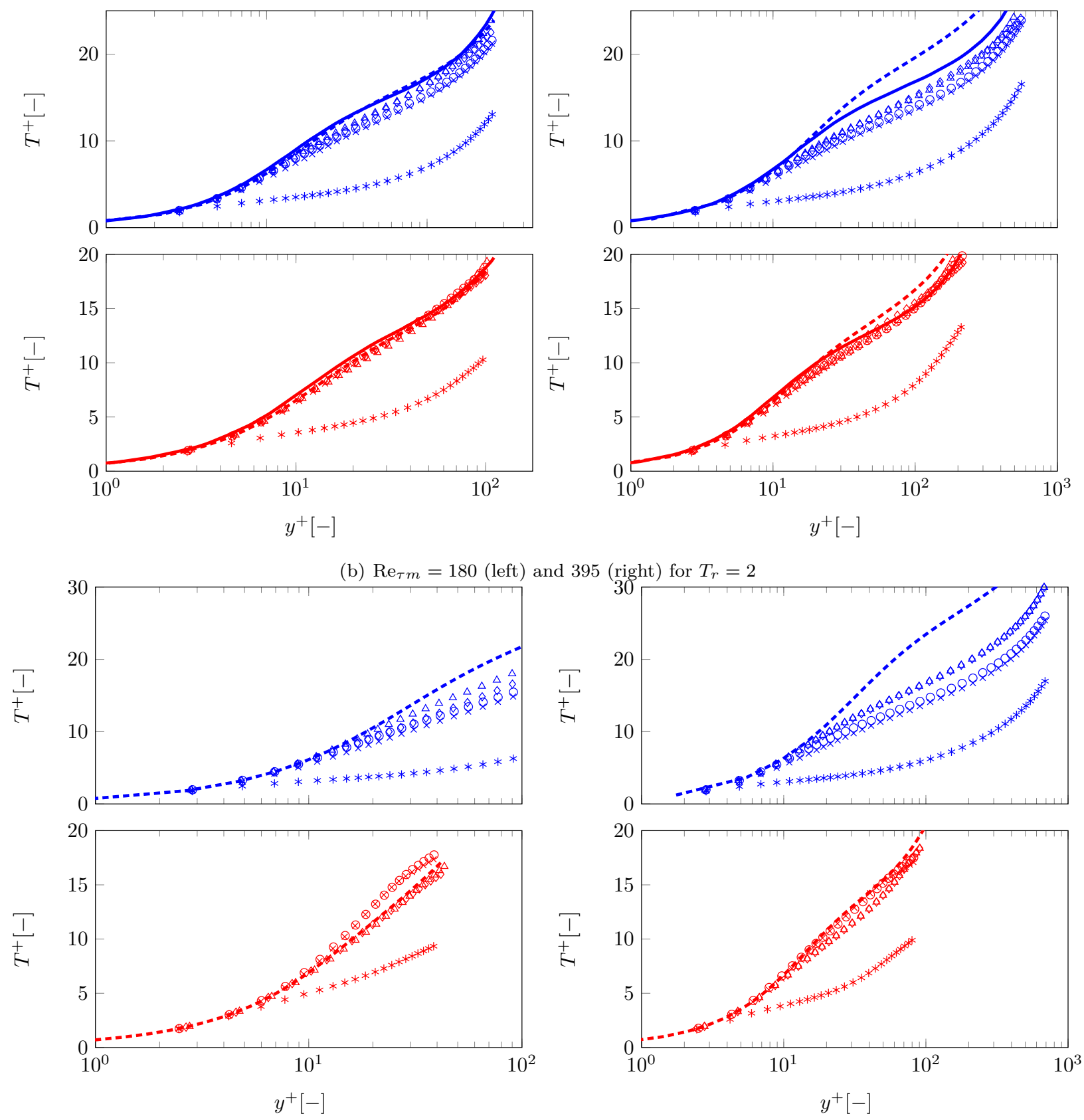

(c) $\operatorname{Re}_{\tau m}=180$ (left) and 395 (right) for $T_{r}=5$

Figure 16 - Non-dimensional temperature $T^{+}$on the hot (red) and cold (blue) walls in function of $y^{+}$ 


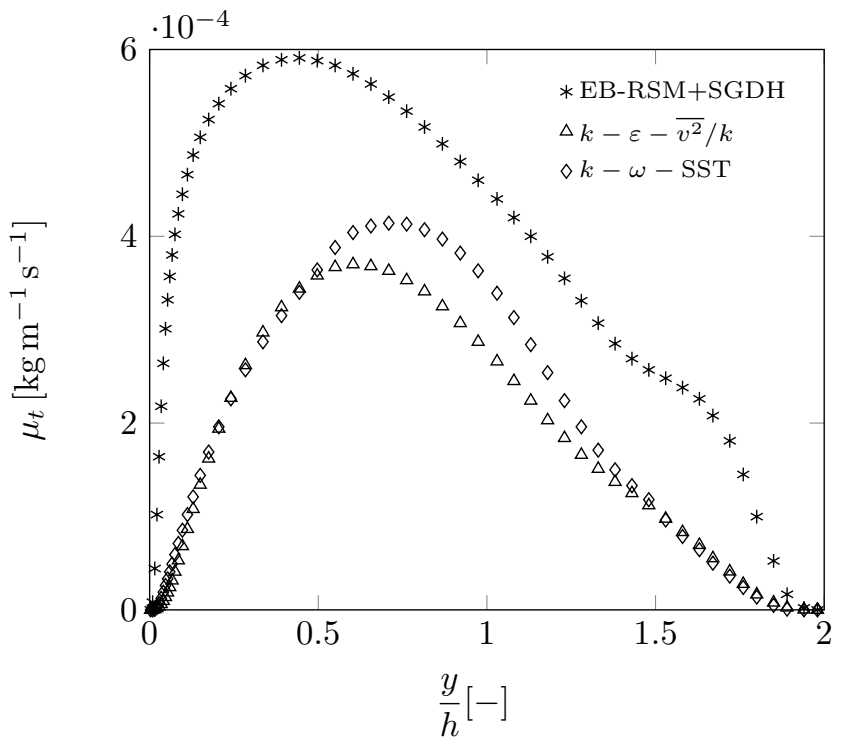

Figure 17 - Comparison of the eddy viscosities for case 180-5. 


\section{Conclusion}

The present paper is concerned with computations of turbulent Poiseuille flows in the presence of a transverse temperature gradient with so-called low-Reynolds number RANS models, which avoid the use of wall functions, namely the EB-RSM and both the $k-\varepsilon-\overline{v^{2}} / k$ and $k-\omega-\mathrm{SST}$ eddy-viscosity models. The objective is manifold: i) testing the capabilities of RANS models to reproduce the main features of the flow predicted by DNS and/or LES computations in such situations, ii) comparing Reynolds-stress and eddy-viscosity models, iii) studying the influence of the model for the turbulent heat flux associated with the Reynolds-stress model (SGDH, GGDH or DFM). Comparisons are performed for two friction Reynolds numbers and five temperature ratios.

The first conclusion concerns the capability of the RANS models to reproduce the main characteristics of the flow, especially the asymmetric velocity profiles. Due to the temperature gradient, the molecular viscosity and the density of the fluid strongly vary between the cold and the hot wall, and the turbulent viscosity exhibits significantly asymmetric profiles. In contrast with the laminar case, since turbulent transport is dominant, the presence of a temperature gradient leads to a shift of the maximum velocity towards the hot side, in accordance with available DNS results. Furthermore, RANS computations are able to predict the departure of the velocity profiles in wall units from the standard law-of-the-wall. Is is noted that the particular choice of the turbulent heat flux model associated to the EB-RSM does not significantly affect the prediction of these velocity profiles. The tendency of the flow to relaminarize, observed in the literature when the temperature ratio increases, is also obtained.

The influence of the turbulence model is in contrast very influential for the prediction of the temperature profiles. When associated with the EB-RSM, the generalized gradient diffusion hypothesis (GGDH) and differential flux model (DFM) for the turbulent heat flux give very similar results, but the simple gradient diffusion hypothesis (SGDH) fails to reproduce the temperature field correctly. All models produce satisfactory results for temperature ratios up to $T_{r}=2$, for which DNS data are available. For $T_{r}=5$, the temperature profiles predicted by the different models exhibit significant discrepancies, especially on the cold side, and a clear distinction appear among Reynolds-stress and eddy-viscosity models.

Eventually, the present study highlights that low-Reynolds number RANS models have good prospects for the computation of industrial flows where strong transverse temperature gradients are involved. It is unfortunately not possible at the present time to discriminate between the Reynolds-stress and eddy-viscosity models, which illustrates the necessity for further DNS studies for high friction Reynolds number and/or temperature ratios.

\section{Acknowledgments}

The authors want to warmly acknowledge Dr. Auléry, Dr. Toutant and Pr. Bataille for providing the DNS data used for the comparisons of the non-dimensional profiles. 


\section{A Characteristics of the various cases for each RANS models}

Table 3 - Characteristics of the $k-\varepsilon-\overline{v^{2}} / k$ calculations

\begin{tabular}{|c|c|c|c|c|c|c|c|c|c|}
\hline $\begin{array}{c}\text { Case } \\
\beta\end{array}$ & $\begin{array}{l}T_{2} \\
T_{1}\end{array}$ & $T_{r}$ & $\begin{array}{l}\operatorname{Re}_{\tau, 2} \\
\operatorname{Re}_{\tau m} \\
\operatorname{Re}_{\tau, 1} \\
\end{array}$ & $\mathrm{Re}_{b}$ & $\begin{array}{l}U_{\tau, 2} \\
U_{\tau, 1}\end{array}$ & $U_{b}$ & $\begin{array}{l}T_{\tau, 2} \\
T_{\tau, 1}\end{array}$ & $T_{m}$ & $\begin{array}{l}y_{2}^{+} \\
y_{1}^{+}\end{array}$ \\
\hline & 293 & & 180 & 2793 & 0.180 & & $\varnothing$ & & 0.900 \\
\hline $180-1$ & & 1 & 180 & 2800 & & 2.81 & & 293 & \\
\hline \multirow[t]{2}{*}{2.62} & 293 & & 180 & 2860 & 0.180 & 2.90 & $\varnothing$ & 293 & 0.900 \\
\hline & 296 & & 179 & 2855 & 0.183 & & 0.0687 & & 0.894 \\
\hline $180-1.01$ & & 1.01 & 180 & 2800 & & 2.84 & & 295 & \\
\hline \multirow[t]{2}{*}{2.65} & 293 & & 181 & 2830 & 0.182 & 3.11 & 0.0677 & 293 & 0.906 \\
\hline & 586 & & 99 & & 0.341 & & 8.2 & & 0.899 \\
\hline $180-2$ & & 2 & 180 & 2322 & & 4.56 & & 431 & \\
\hline 5 & 293 & & 261 & 2380 & 0.256 & 4.93 & 5.4 & 442 & 0.902 \\
\hline & 1176 & & 55 & & 0.555 & & 29.8 & & 0.899 \\
\hline \multirow[t]{2}{*}{$180-4$} & & 4 & 180 & 1439 & & 6.21 & & 691 & \\
\hline & 293 & & 306 & & 0.307 & & 12.1 & & 0.873 \\
\hline & 1465 & & 44 & & 0.638 & & 41.6 & & 0.888 \\
\hline $180-5$ & & 5 & 180 & 1175 & & 6.72 & & 821 & \\
\hline \multirow[t]{2}{*}{7.4} & 293 & & 316 & 1160 & 0.318 & 7.53 & 16.13 & 890 & 0.904 \\
\hline & 293 & & 395 & 6881 & 0.396 & & $\varnothing$ & & 0.900 \\
\hline $395-1$ & & 1 & 395 & 6915 & & 6.94 & & 293 & \\
\hline \multirow[t]{2}{*}{12.62} & 293 & & 395 & 7630 & 0.396 & 7.53 & $\varnothing$ & 293 & 0.900 \\
\hline & 313 & & 376 & & 0.425 & & 0.424 & & 0.859 \\
\hline $395-1.07$ & & 1.07 & 380 & 6904 & & 7.35 & & 303 & \\
\hline \multirow[t]{2}{*}{12.78} & 293 & & 413 & & 0.415 & & 0.405 & & 0.942 \\
\hline & 586 & & 225 & & 0.742 & & 7.54 & & 0.897 \\
\hline $395-2$ & & 2 & 395 & 5806 & & 11.27 & & 428 & \\
\hline 24 & 293 & & 565 & 5860 & 0.567 & 12.6 & 4.87 & 460 & 0.901 \\
\hline & 1176 & & 116 & & 1.17 & & 27.7 & & 0.835 \\
\hline \multirow[t]{2}{*}{$395-4$} & & 4 & 395 & 3712 & & 15.3 & & 671 & \\
\hline & 293 & & 675 & & 0.677 & & 11.7 & & 0.886 \\
\hline & 1465 & & 93 & & 1.34 & & 39.2 & & 0.892 \\
\hline $395-5$ & & 5 & 395 & 3068 & & 16.49 & & 790 & \\
\hline 34.4 & 293 & & 697 & 2957 & 0.699 & 20.7 & 14.5 & 962 & 0.901 \\
\hline
\end{tabular}


Table 4 - Characteristics of the $k-\omega-$ SST calculations

\begin{tabular}{|c|c|c|c|c|c|c|c|c|c|}
\hline $\begin{array}{c}\text { Case } \\
\beta \\
\end{array}$ & $\begin{array}{l}T_{2} \\
T_{1}\end{array}$ & $T_{r}$ & $\begin{array}{l}\operatorname{Re}_{\tau, 2} \\
\operatorname{Re}_{\tau m} \\
\operatorname{Re}_{\tau, 1} \\
\end{array}$ & $\operatorname{Re}_{b}$ & $\begin{array}{c}U_{\tau, 2} \\
U_{\tau, 1}\end{array}$ & $U_{b}$ & $\begin{array}{l}T_{\tau, 2} \\
T_{\tau, 1}\end{array}$ & $T_{m}$ & $\begin{array}{l}y_{2}^{+} \\
y_{1}^{+}\end{array}$ \\
\hline & 293 & & 180 & 2793 & 0.181 & & $\varnothing$ & & 0.900 \\
\hline $180-1$ & & 1 & 180 & 2825 & & 2.84 & & 293 & \\
\hline \multirow[t]{2}{*}{2.62} & 293 & & 180 & 2860 & 0.181 & 2.90 & $\varnothing$ & 293 & 0.900 \\
\hline & 296 & & 179 & 2855 & 0.183 & & 0.0735 & & 0.894 \\
\hline $180-1.01$ & & 1.01 & 180 & 2829 & & 2.86 & & 295 & \\
\hline \multirow{2}{*}{2.65} & 293 & & 181 & 2830 & 0.182 & 3.11 & 0.0740 & 293 & 0.907 \\
\hline & 586 & & 102 & & 0.337 & & 8.9 & & 0.886 \\
\hline $180-2$ & & 2 & 180 & 2329 & & 4.53 & & 429 & \\
\hline \multirow[t]{2}{*}{5} & 293 & & 257 & 2380 & 0.258 & 4.93 & 5.7 & 442 & 0.907 \\
\hline & 1176 & & 53 & & 0.535 & & 32.0 & & 1.057 \\
\hline \multirow[t]{3}{*}{$180-4$} & & 4 & 180 & 1415 & & 5.89 & & 676 & \\
\hline & 293 & & 307 & & 0.308 & & 13.4 & & 0.877 \\
\hline & 1465 & & 42 & & 0.615 & & 44.9 & & 0.855 \\
\hline $180-5$ & & 5 & 180 & 1137 & & 6.26 & & 802 & \\
\hline \multirow[t]{2}{*}{7.4} & 293 & & 317 & 1160 & 0.318 & 7.53 & 16.6 & 890 & 0.907 \\
\hline & 293 & & 395 & 6881 & 0.397 & & $\varnothing$ & & 0.901 \\
\hline $395-1$ & & 1 & 395 & 7040 & & 7.06 & & 293 & \\
\hline \multirow[t]{2}{*}{12.62} & 293 & & 395 & 7630 & 0.397 & 7.53 & $\varnothing$ & 293 & 0.901 \\
\hline & 313 & & 377 & & 0.425 & & 0.469 & & 0.859 \\
\hline $395-1.07$ & & 1.07 & 395 & 7019 & & 7.47 & & 303 & \\
\hline \multirow[t]{2}{*}{12.78} & 293 & & 413 & & 0.414 & & 0.450 & & 0.941 \\
\hline & 586 & & 223 & & 0.735 & & 8.40 & & 0.888 \\
\hline $395-2$ & & 2 & 395 & 5890 & & 11.37 & & 427 & \\
\hline 24 & 293 & & 567 & 5860 & 0.569 & 12.6 & 5.33 & 460 & 0.905 \\
\hline & 1176 & & 112 & & 1.14 & & 30.8 & & 1.080 \\
\hline \multirow[t]{2}{*}{$395-4$} & & 4 & 395 & 3742 & & 15.0 & & 660 & \\
\hline & 293 & & 678 & & 0.680 & & 12.5 & & 0.876 \\
\hline & 1465 & & 90 & & 1.29 & & 43.4 & & 0.862 \\
\hline $395-5$ & & 5 & 395 & 3083 & & 16.0 & & 773 & \\
\hline 34.4 & 293 & & 700 & 2957 & 0.703 & 20.7 & 15.4 & 962 & 0.906 \\
\hline
\end{tabular}


Table 5 - Characteristics of the EB-RSM+SGDH calculations

\begin{tabular}{|c|c|c|c|c|c|c|c|c|c|}
\hline $\begin{array}{c}\text { Case } \\
\beta \\
\end{array}$ & $T_{2}$ & $T_{r}$ & $\begin{array}{l}\operatorname{Re}_{\tau, 2} \\
\operatorname{Re}_{\tau m} \\
\operatorname{Re}_{\tau, 1} \\
\end{array}$ & $\operatorname{Re}_{b}$ & $\begin{array}{l}U_{\tau, 2} \\
U_{\tau, 1}\end{array}$ & $U_{b}$ & $\begin{array}{l}T_{\tau, 2} \\
T_{\tau, 1}\end{array}$ & $T_{m}$ & $\begin{array}{l}y_{2}^{+} \\
y_{1}^{+}\end{array}$ \\
\hline \multirow{3}{*}{$180-1$} & 293 & \multirow{3}{*}{1} & 180 & 2793 & 0.181 & & $\varnothing$ & & 0.900 \\
\hline & & & 180 & 2867 & & 2.88 & & 293 & \\
\hline & 293 & & 180 & 2860 & 0.181 & 2.90 & $\varnothing$ & 293 & 0.900 \\
\hline \multirow{3}{*}{$180-1.01$} & 296 & \multirow{3}{*}{1.01} & 179 & 2855 & 0.183 & & 0.121 & & 0.894 \\
\hline & & & 180 & 2871 & & 2.91 & & 295 & \\
\hline & 293 & & 181 & 2830 & 0.182 & 3.11 & 0.121 & 293 & 0.907 \\
\hline \multirow{3}{*}{$180-2$} & 586 & \multirow{3}{*}{ 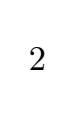 } & 99 & & 0.326 & & 15.81 & & 0.858 \\
\hline & & & 180 & 2376 & & 4.57 & & 426 & \\
\hline & 293 & & 262 & 2380 & 0.262 & 4.9 & 9.56 & 442 & 0.923 \\
\hline \multirow{3}{*}{$180-4$} & 1176 & & 49 & & 0.499 & & 60.3 & & 0.807 \\
\hline & & & 180 & 1529 & & 5.79 & & 638 & \\
\hline & 293 & & 311 & & 0.313 & & 22.8 & & 0.890 \\
\hline \multirow{3}{*}{$180-5$} & 1465 & & 40 & & 0.569 & & 83.7 & & 0.791 \\
\hline & & & 180 & 1282 & & 6.04 & & 728 & \\
\hline & 293 & & 320 & 1160 & 0.321 & 7.5 & 27.8 & 890 & 0.915 \\
\hline \multirow{3}{*}{$395-1$} & 293 & & 395 & 6881 & 0.396 & & $\varnothing$ & & 0.900 \\
\hline & & & 395 & 7000 & & 7.02 & & 293 & \\
\hline & 293 & & 395 & 7630 & 0.396 & 7.53 & $\varnothing$ & 293 & 0.900 \\
\hline \multirow{3}{*}{$395-1.07$} & 313 & & 376 & & 0.424 & & 0.644 & & 0.857 \\
\hline & & \multirow[t]{2}{*}{1.07} & 395 & 7000 & & 7.45 & & 303 & \\
\hline & 293 & & 415 & & 0.416 & & 0.614 & & 0.945 \\
\hline \multirow{3}{*}{$395-2$} & 586 & & 217 & & 0.712 & & 12.3 & & 0.862 \\
\hline & & & 395 & 5797 & & 11. & & 427 & \\
\hline & 293 & & 573 & 5860 & 0.575 & 12.6 & 7.47 & 460 & 0.915 \\
\hline \multirow{3}{*}{$395-4$} & 1176 & & 105 & & 1.06 & & 51.6 & & 0.753 \\
\hline & & & 395 & 3635 & & 14.3 & & 653 & \\
\hline & 293 & & 684 & & 0.687 & & 19.0 & & 0.900 \\
\hline \multirow{3}{*}{$395-5$} & 1465 & & 82 & & 1.18 & & 75.7 & & 0.786 \\
\hline & & & 395 & 3033 & & 15.07 & & 753 & \\
\hline & 293 & & 707 & 2957 & 0.710 & 20.7 & 23.8 & 962 & 0.914 \\
\hline
\end{tabular}


Table 6 - Characteristics of the EB-RSM+GGDH calculations

\begin{tabular}{|c|c|c|c|c|c|c|c|c|c|}
\hline $\begin{array}{c}\text { Case } \\
\beta \\
\end{array}$ & $T_{2}$ & $T_{r}$ & $\begin{array}{l}\operatorname{Re}_{\tau, 2} \\
\operatorname{Re}_{\tau m} \\
\operatorname{Re}_{\tau, 1}\end{array}$ & $\operatorname{Re}_{b}$ & $\begin{array}{l}U_{\tau, 2} \\
U_{\tau, 1}\end{array}$ & $U_{b}$ & $\begin{array}{l}T_{\tau, 2} \\
T_{\tau, 1}\end{array}$ & $T_{m}$ & $\begin{array}{l}y_{2}^{+} \\
y_{1}^{+}\end{array}$ \\
\hline & 293 & & 180 & 2793 & $\overline{0.180}$ & & $\varnothing$ & & 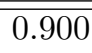 \\
\hline $180-1$ & & 1 & 180 & 2866 & & 2.88 & & 293 & \\
\hline \multirow[t]{2}{*}{2.62} & 293 & & 180 & 2860 & 0.180 & 2.90 & $\varnothing$ & 293 & 0.900 \\
\hline & 296 & & 179 & 2855 & 0.183 & & 0.075 & & 0.894 \\
\hline $180-1.01$ & & 1.01 & 180 & 2869 & & 2.91 & & 295 & \\
\hline \multirow[t]{2}{*}{2.65} & 293 & & 181 & 2830 & 0.182 & 3.11 & 0.121 & 293 & 0.907 \\
\hline & 586 & & 100 & & 0.330 & & 9.91 & & 0.867 \\
\hline $180-2$ & & 2 & 180 & 2410 & & 4.24 & & 424 & \\
\hline \multirow[t]{2}{*}{4.89} & 293 & & 260 & 2380 & 0.261 & 4.9 & 5.65 & 442 & 0.919 \\
\hline & 1176 & & 41 & & 0.597 & & & & 0.680 \\
\hline $180-4$ & & 4 & 180 & 1516 & & 5.85 & & 645 & \\
\hline \multirow[t]{2}{*}{4.89} & 293 & & 310 & & 0.312 & & 12.54 & & 0.888 \\
\hline & 1465 & & 40 & & 0.571 & & 44.6 & & 0.917 \\
\hline $180-5$ & & 5 & 180 & 1224 & & 6.1 & & 758 & \\
\hline \multirow[t]{2}{*}{6.9} & 293 & & 321 & 1160 & 0.322 & 7.5 & 15.3 & 890 & 0.917 \\
\hline & 293 & & 395 & 6881 & 0.396 & & $\varnothing$ & & 0.900 \\
\hline $395-1$ & & 1 & 395 & 7000 & & 7.03 & & 293 & \\
\hline \multirow[t]{2}{*}{12.62} & 293 & & 395 & 7630 & 0.396 & 7.53 & $\varnothing$ & 293 & 0.900 \\
\hline & 313 & & 376 & & 0.424 & & 0.424 & & 0.856 \\
\hline $395-1.07$ & & 1.07 & 395 & 7000 & & 7.45 & & 303 & \\
\hline \multirow[t]{2}{*}{12.78} & 293 & & 414 & & 0.416 & & 0.438 & & 0.943 \\
\hline & 586 & & 220 & & 0.724 & & 8.34 & & 0.875 \\
\hline $395-2$ & & 2 & 395 & 5916 & & 11.29 & & 424 & \\
\hline \multirow[t]{2}{*}{23.6} & 293 & & 571 & 5860 & 0.572 & 12.6 & 5.19 & 460 & 0.911 \\
\hline & 1176 & & 108 & & 1.09 & & 31.4 & & 0.775 \\
\hline $395-4$ & & 4 & 395 & 3864 & & 14.7 & & 639 & \\
\hline \multirow{2}{*}{23.6} & 293 & & 693 & & 0.685 & & 12.1 & & 0.897 \\
\hline & 1465 & & 84 & & 1.21 & & 44.5 & & 0.808 \\
\hline $395-5$ & & 5 & 395 & 3243 & & 15. & & 735 & \\
\hline 32.1 & 293 & & 706 & 2957 & 0.709 & 20.7 & 14.7 & 962 & 0.913 \\
\hline
\end{tabular}


Table 7 - Characteristics of the EB-RSM+DFM calculations

\begin{tabular}{|c|c|c|c|c|c|c|c|c|c|}
\hline $\begin{array}{c}\text { Case } \\
\beta \\
\end{array}$ & $T_{2}$ & $T_{r}$ & $\begin{array}{l}\operatorname{Re}_{\tau, 2} \\
\operatorname{Re}_{\tau m} \\
\operatorname{Re}_{\tau, 1} \\
\end{array}$ & $\operatorname{Re}_{b}$ & $\begin{array}{l}U_{\tau, 2} \\
U_{\tau, 1}\end{array}$ & $U_{b}$ & $\begin{array}{l}T_{\tau, 2} \\
T_{\tau, 1}\end{array}$ & $T_{m}$ & $\begin{array}{l}y_{2}^{+} \\
y_{1}^{+}\end{array}$ \\
\hline \multirow{3}{*}{$180-1$} & 293 & \multirow{3}{*}{1} & 180 & 2793 & 0.181 & & $\varnothing$ & & 0.900 \\
\hline & & & 180 & 2867 & & 2.87 & & 293 & \\
\hline & 293 & & 180 & 2860 & 0.181 & 2.90 & $\varnothing$ & 293 & 0.900 \\
\hline \multirow{3}{*}{$180-1.01$} & 296 & \multirow{3}{*}{1.01} & 179 & 2855 & 0.182 & & 0.078 & & 0.893 \\
\hline & & & 180 & 2867 & & 2.90 & & 295 & \\
\hline & 293 & & 181 & 2830 & 0.182 & 3.11 & 0.075 & 293 & 0.906 \\
\hline \multirow{3}{*}{$180-2$} & 586 & \multirow{3}{*}{ 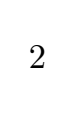 } & 100 & & 0.329 & & 9.32 & & 0.865 \\
\hline & & & 180 & 2417 & & 4.60 & & 423 & \\
\hline & 293 & & 261 & 2380 & 0.262 & 4.9 & 5.77 & 442 & 0.920 \\
\hline \multirow{3}{*}{$180-4$} & 1176 & & 49 & & 0.498 & & 32.9 & & 0.806 \\
\hline & & & 180 & 1519 & & 5.80 & & 642 & \\
\hline & 293 & & 310 & & 0.311 & & 12.8 & & 0.886 \\
\hline \multirow{3}{*}{$180-5$} & 1465 & & 40 & & 0.568 & & 45.6 & & 0.790 \\
\hline & & & 180 & 1223 & & 6.08 & & 753 & \\
\hline & 293 & & 320 & 1160 & 0.321 & 7.5 & 15.6 & 890 & 0.914 \\
\hline \multirow{3}{*}{$395-1$} & 293 & & 395 & 6881 & 0.396 & & $\varnothing$ & & 0.900 \\
\hline & & & 395 & 7000 & & 7.02 & & 293 & \\
\hline & 293 & & 395 & 7630 & 0.396 & 7.53 & $\varnothing$ & 293 & 0.900 \\
\hline \multirow{3}{*}{$395-1.07$} & 313 & & 376 & & 0.424 & & 0.470 & & 0.858 \\
\hline & & \multirow[t]{2}{*}{1.07} & 395 & 6999 & & 7.45 & & 303 & \\
\hline & 293 & & 414 & & 0.415 & & 0.450 & & 0.943 \\
\hline \multirow{3}{*}{$395-2$} & 586 & & 219 & & 0.722 & & 8.57 & & 0.873 \\
\hline & & & 395 & 5915 & & 11.25 & & 423 & \\
\hline & 293 & & 569 & 5860 & 0.572 & 12.6 & 5.32 & 460 & 0.909 \\
\hline \multirow{3}{*}{$395-4$} & 1176 & & 107 & & 1.08 & & 32.2 & & 0.771 \\
\hline & & & 395 & 3889 & & 14.6 & & 634 & \\
\hline & 293 & & 682 & & 0.685 & & 12.4 & & 0.897 \\
\hline \multirow{3}{*}{$395-5$} & 1465 & & 84 & & 1.21 & & 45.6 & & 0.803 \\
\hline & & & 395 & 3269 & & 15.37 & & 728 & \\
\hline & 293 & & 705 & 2957 & 0.705 & 20.7 & 15.4 & 962 & 0.912 \\
\hline
\end{tabular}




\section{B EB-RSM equations}

$$
\begin{aligned}
\phi_{i j} & =\left(1-\alpha^{3}\right) \phi_{i j}^{w}+\alpha^{3} \phi_{i j}^{h} \\
\varepsilon_{i j} & =\left(1-\alpha^{3}\right) \varepsilon_{i j}^{w}+\alpha^{3} \varepsilon_{i j}^{h} \\
\mathcal{L} & =C_{L} \max \left(\frac{k^{3 / 2}}{\varepsilon} ; C_{\eta} \frac{\nu^{3 / 4}}{\varepsilon^{1 / 4}}\right) \\
\mathbb{T} & =\max \left(\frac{k}{\varepsilon} ; C_{T} \sqrt{\frac{\nu}{\varepsilon}}\right) \\
C_{\varepsilon 1}^{\prime} & =C_{\varepsilon 1}\left(1 .+A_{1}\left(1-\alpha^{3}\right) \frac{\mathcal{P}}{\varepsilon}\right) \\
\mathbf{n} & =\frac{\nabla \alpha}{\|\nabla \alpha\|} \\
\phi_{i j}^{h} & =-\left(C_{1} \varepsilon+C_{2}^{\prime} \mathcal{P}\right) a_{i j}+\left(C_{3}-C_{3}^{\prime}\left(a_{k l} a_{k l}\right)^{0,5}\right) k S_{i j}+C_{4} k\left(a_{i k} S_{j k}+a_{j k} S i k-\frac{2}{3} a_{k l} S_{k l} \delta_{i j}\right) \\
& +C_{5}\left(a_{i k} \Omega_{j k}+a_{j k} \Omega_{i k}\right) \\
\phi_{i j}^{w} & =-5 \frac{\varepsilon}{k}\left[\tau_{i k} n_{j} n_{k}+\tau_{j k} n_{i} n_{k}-\frac{1}{2} \tau_{k l} n_{k} n_{l}\left(n_{i} n_{j}+\delta_{i j}\right)\right] \\
\tau_{i j} & =\overline{U_{i}^{\prime} U_{j}^{\prime}} \\
\varepsilon_{i j}^{w} & =\frac{\overline{U_{i}^{\prime} U_{j}^{\prime}}}{k} \varepsilon \\
\varepsilon_{i j}^{h} & =\frac{2}{3} \varepsilon \delta_{i j} \\
a_{i j} & =\frac{\overline{U_{i}^{\prime} U_{j}^{\prime}}}{k}-\frac{2}{3} \delta_{i j} \\
\Omega_{i j} & =\frac{1}{2}\left(\frac{\partial \overline{U_{i}}}{\partial x_{j}}-\frac{\partial \overline{U_{j}}}{\partial x_{i}}\right) \\
\mathcal{P}_{i j} & =-\overline{U_{i}^{\prime} U_{k}^{\prime}} \frac{\partial \overline{U_{j}}}{\partial x_{k}}-\overline{U_{j}^{\prime} U_{k}^{\prime}} \frac{\partial \overline{U_{i}}}{\partial x_{k}} \\
D_{i j}^{T} & =\frac{\partial}{\partial x_{l}}\left(\rho \frac{C_{\mu}}{\sigma_{k}} \overline{U_{l}^{\prime} U_{m}^{\prime}} \mathbb{T} \frac{\partial U_{i}^{\prime} U_{j}^{\prime}}{\partial x_{m}}\right) \\
&
\end{aligned}
$$

And:

\begin{tabular}{ccccccccccccc}
\hline$C_{1}$ & $C_{2}^{\prime}$ & $C_{3}$ & $C_{3}^{\prime}$ & $C_{4}$ & $C_{5}$ & $C_{L}$ & $C_{\eta}$ & $C_{T}$ & $C_{\varepsilon 1}$ & $A_{1}$ & $C_{\varepsilon 2}$ & $\sigma_{\varepsilon}$ \\
\hline 1.7 & 0.9 & 0.8 & 0.65 & 0.625 & 0.2 & 0.122 & 80 & 6 & 1.44 & 0.1 & 1.83 & 1.15 \\
\hline
\end{tabular}

Table 8 - EB-RSM constants 
C $\quad k-\varepsilon-\overline{v^{2}} / k$ equations

$$
\begin{aligned}
f_{w} & =-\frac{\varepsilon}{2} \frac{\varphi}{k} \\
f_{h} & =-\frac{1}{\mathbb{T}}\left(C_{1}-1+C_{2} \frac{\mathcal{P}}{\varepsilon}\right)\left(\varphi-\frac{2}{3}\right) \\
C_{\varepsilon 2}^{*} & =C_{\varepsilon 2}+\alpha^{3}\left(C_{\varepsilon 4}-C_{\varepsilon 2}\right) \tanh \left(\left|\frac{\frac{\partial}{\partial x_{i}}\left(\frac{\nu_{t}}{\sigma_{k}} \frac{\partial k}{\partial x_{i}}\right)}{\varepsilon}\right|^{3 / 2}\right) \\
\mathcal{L} & =\sqrt{C_{L}^{2}\left(\frac{k^{3}}{\varepsilon^{2}}+C_{\eta}^{2} \frac{\nu^{3 / 2}}{\varepsilon^{1 / 2}}\right)}
\end{aligned}
$$

And:

\begin{tabular}{ccccccccccccc}
\hline$C_{\varepsilon 1}$ & $C_{\varepsilon 2}$ & $C_{\varepsilon 3}$ & $C_{\varepsilon 4}$ & $\sigma_{k}$ & $\sigma_{\varepsilon}$ & $C_{\mu}$ & $C_{T}$ & $C_{L}$ & $C_{\eta}$ & $C_{1}$ & $C_{2}$ & $\sigma_{\varphi}$ \\
\hline 1.44 & 1.83 & 2.3 & 0.4 & 1 & 1.5 & 0.22 & 4 & 0.164 & 75 & 1.7 & 0.9 & 1 \\
\hline
\end{tabular}

Table $9-k-\varepsilon-\overline{v^{2}} / k$ constants 


\section{D $\quad k-\omega-$ SST equations}

$$
\begin{aligned}
\frac{\partial \rho k}{\partial t}+\overline{U_{j}} \frac{\partial \rho k}{\partial x_{j}} & =\rho \mathcal{P}_{k}-\beta^{*} \rho \omega k+\frac{\partial}{\partial x_{j}}\left[\left(\mu+\frac{\mu_{t}}{\sigma_{k}}\right) \frac{\partial k}{\partial x_{j}}\right] \\
\frac{\partial \rho \omega}{\partial t}+\overline{U_{j}} \frac{\partial \rho \omega}{\partial x_{j}} & =\frac{\gamma}{\nu_{t}} \rho \mathcal{P}_{k}-\beta \rho \omega^{2}+\frac{\partial}{\partial x_{j}}\left[\left(\mu+\frac{\mu_{t}}{\sigma_{\omega}}\right) \frac{\partial \omega}{\partial x_{j}}\right]+2\left(1-F_{1}\right) \rho \sigma_{\omega} \frac{1}{\omega} \frac{\partial k}{\partial x_{j}} \frac{\partial \omega}{\partial x_{j}}
\end{aligned}
$$

With :

$$
\begin{aligned}
\nu_{t} & =\frac{a_{1} k}{\max \left(a_{1} \omega, S F_{2}\right)} \\
\mathcal{P}_{k} & =\min \left(-\tau_{i j} \frac{\partial \overline{U_{i}}}{\partial x_{j}}, 10 \beta^{*} k \omega\right) \\
F_{1} & =\tanh \left[\left[\min \left[\max \left(\frac{\sqrt{k}}{\beta^{*} \omega y}, \frac{500 \nu}{y^{2} \omega}\right), \frac{4 \rho \sigma_{\omega_{2}} k}{\mathrm{CD}_{k \omega} y^{2}}\right]\right]^{4}\right] \\
F_{2} & =\tanh \left[\left[\max \left(\frac{2 \sqrt{k}}{\beta^{*} \omega y}, \frac{500 \nu}{y^{2} \omega}\right)\right]^{2}\right] \\
\mathrm{CD}_{k \omega} & =\max \left(2 \rho \sigma_{\omega 2} \frac{1}{\omega} \frac{\partial k}{\partial x_{i}} \frac{\partial \omega}{\partial x_{i}}, 10^{-10}\right)
\end{aligned}
$$

The constant $\phi$ are computed from the constants $\phi_{1}$ and $\phi_{2}$ :

Table $10-k-\omega-S S T$ constants

\begin{tabular}{ccccc}
\hline & $\sigma_{k}$ & $\sigma_{\omega}$ & $\beta$ & $\gamma$ \\
\hline$\phi_{1}$ & 1.1765 & 2.0 & 0.075 & 0.54 \\
$\phi_{2}$ & 1.0 & 1.1682 & 0.0828 & 0.42 \\
\hline
\end{tabular}




\section{E Supplementary turbulent profiles}
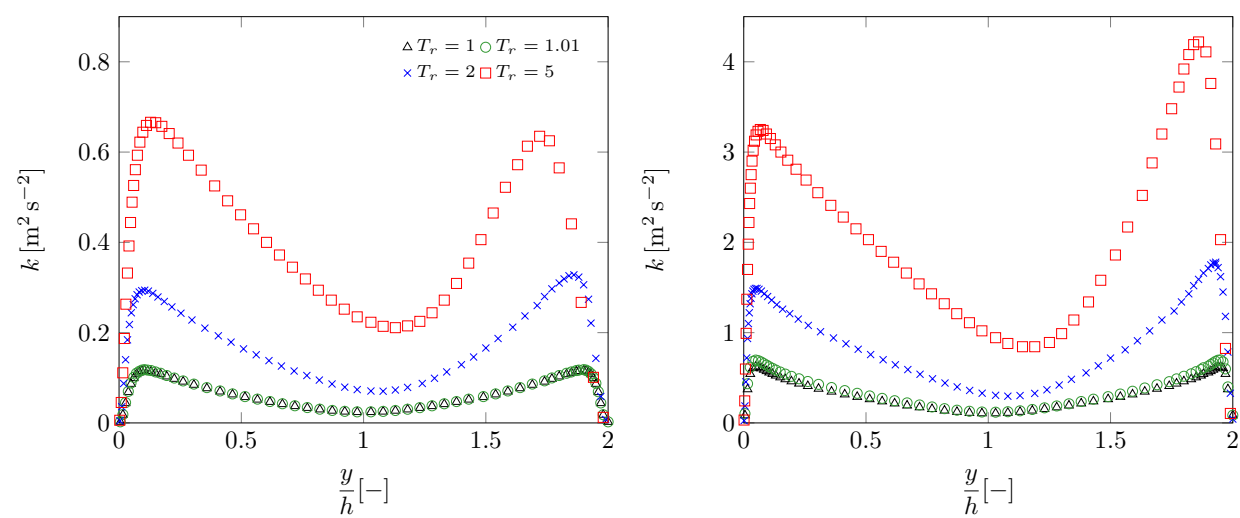

(a) $k-\varepsilon-\overline{v^{2}} / k$
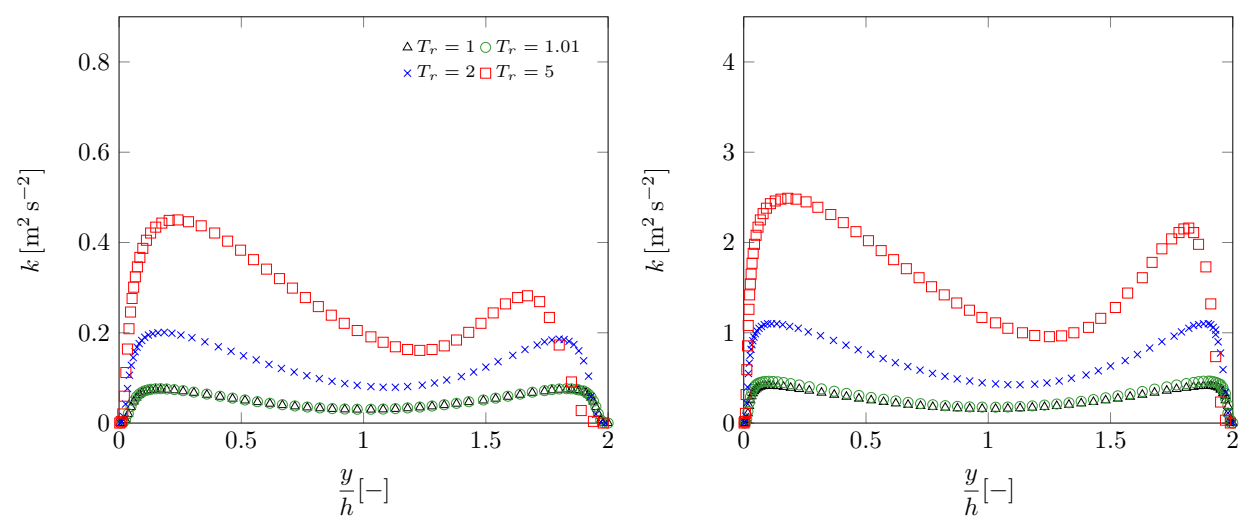

(b) $k-\omega-\mathrm{SST}$
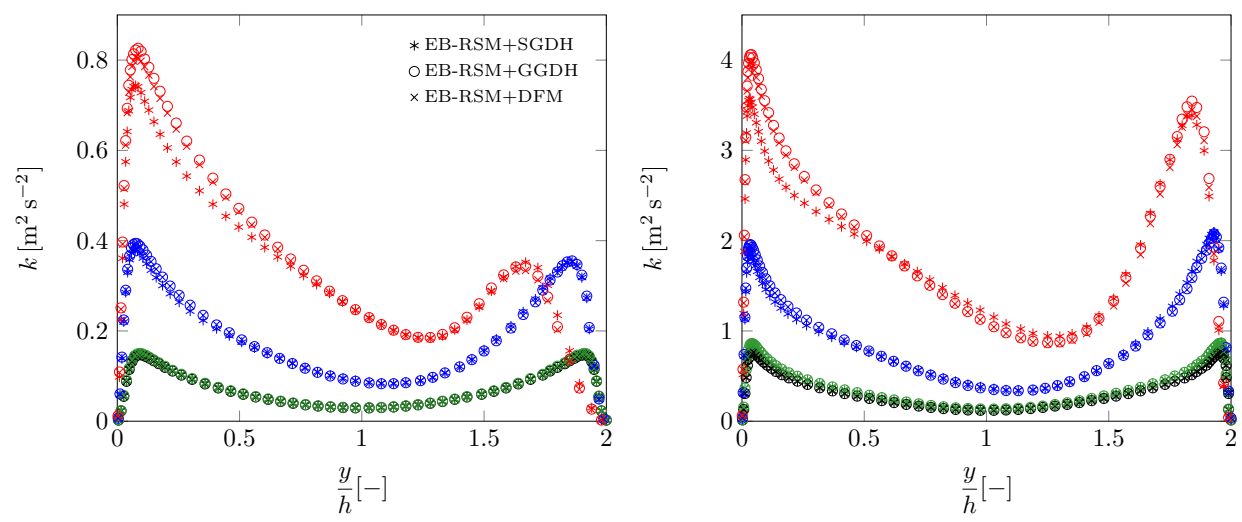

(c) EB-RSM

Figure 18 - Evolution of the turbulent kinetic energy between the two plates at the two turbulent Reynolds numbers $\operatorname{Re}_{\tau m}=180$ (left) and 395 (right) for the four temperature ratios $T_{r}=1$ (black), $1.01 \& 1.07$ (green), 2 (blue) and 5 (red) 

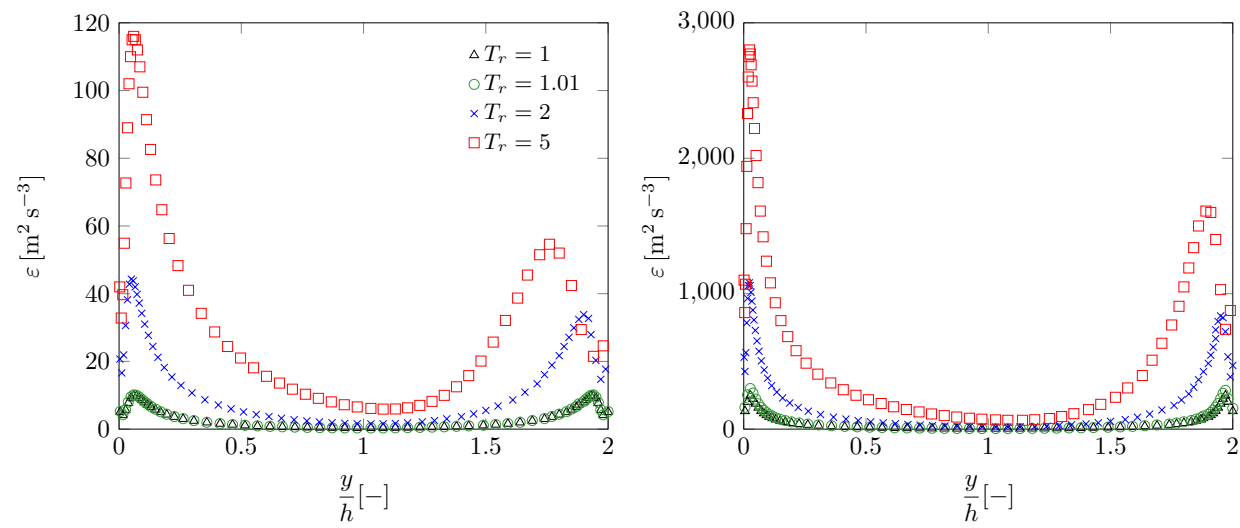

(a) $k-\varepsilon-\overline{v^{2}} / k$
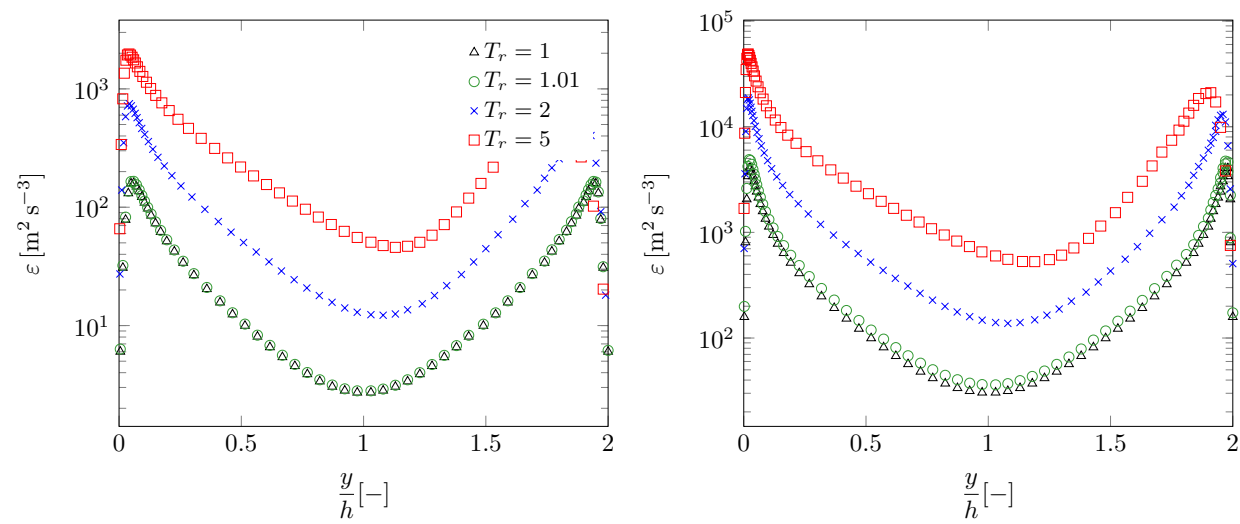

(b) $k-\omega-\mathrm{SST}$
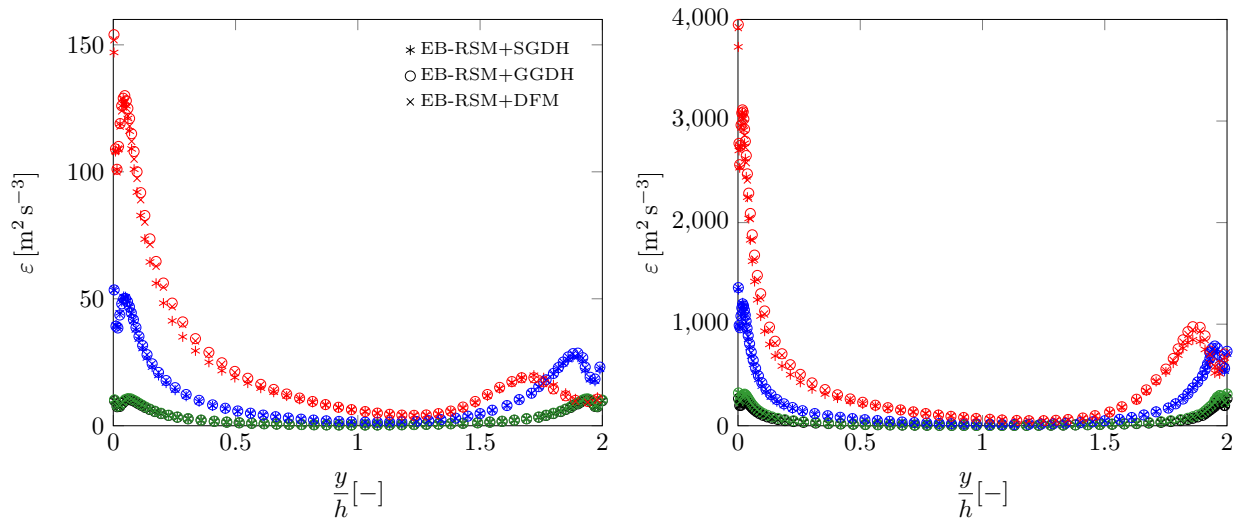

(c) EB-RSM

Figure 19 - Evolution of the turbulent dissipation rate between the two plates at the two turbulent Reynolds numbers $\operatorname{Re}_{\tau m}=180$ (left) and 395 (right) for the four temperature ratios $T_{r}=1$ (black), $1.01 \& 1.07$ (green), 2 (blue) and 5 (red) 


\section{References}

[1] Sylvain Serra, Adrien Toutant, Françoise Bataille, and Ye Zhou. Turbulent kinetic energy spectrum in very anisothermal flows. Physics Letters A, 376:3177-3184, 2012.

[2] S. Paolucci. On the filtering of sound from the Navier-Stokes equations. Sandia National Laboratories Livermore, CA, 1982.

[3] John Kim, Parviz Moin, and Robert Moser. Turbulence statistics in fully developed channel flow at low Reynolds number. Journal of fluid mechanics, 177:133-166, 1987.

[4] Robert D Moser, John Kim, and Nagi N Mansour. Direct numerical simulation of turbulent channel flow up to $\operatorname{Re}_{\tau}=$ 590. Physics of fluids, 11(4):943-945, 1999.

[5] S. Hoyas and J. Jimenez. Scaling of velocity fluctuations in turbulent channels up to $R e_{\tau}=2000$. Phys. Fluids, 18(1), 2006.

[6] M. Lee and R.D. Moser. Direct numerical simulation of turbulent channel flow up to $R e_{\tau} \approx 5200$. J. Fluid Mech., 774:395-415, 2015.

[7] J. Kim and P. Moin. Transport of passive scalars in a turbulent channel flow. In Turbulent Shear Flows 6. Selected Papers from the 6th Int. Symp. Turb. Shear Flow, Toulouse, France, September 7-9, 1987,. Springer-Verlag, 1989.

[8] N. Kasagi, Y. Tomita, and A. Kuroda. Direct numerical simulation of passive scalar field in a turbulent channel flow. J. Heat Transf., 114(3):598-606, 1992.

[9] Hiroshi Kawamura, Hiroyuki Abe, and Yuichi Matsuo. DNS of turbulent heat transfer in channel flow with respect to Reynolds and Prandtl number effects. International Journal of Heat and Fluid Flow, 20(3):196-207, 1999.

[10] Makoto Kozuka, Yohji Seki, and Hiroshi Kawamura. DNS of turbulent heat transfer in a channel flow with a high spatial resolution. International Journal of Heat and Fluid Flow, 30(3):514 - 524, 2009. The Seventh International Symposium on Engineering Turbulence Modelling and Measurements, ETMM7.

[11] Hirofumi Hattori, Shohei Yamada, Masahiro Tanaka, Tomoya Houra, and Yasutaka Nagano. DNS, LES and RANS of turbulent heat transfer in boundary layer with suddenly changing wall thermal conditions. International Journal of Heat and Fluid Flow, 41(Supplement C):34 - 44, 2013. ETMM9.

[12] C. Flageul, S. Benhamadouche, E. Lamballais, and D. Laurence. DNS of turbulent channel flow with conjugate heat transfer: Effect of thermal boundary conditions on the second moments and budgets. Int. J. Heat Fluid Fl., 55:34-44, 2015.

[13] N. Kasagi and M. Nishimura. Direct Numerical Simulation of Combined Forced and Natural Turbulent Convection in a Vertical Plane Channel. Int. J. Heat Fluid Fl., 18(1):88-99, 1997.

[14] R. Boudjemadi, V. Maupu, D. Laurence, and P. Le Quéré. Budgets of turbulent stresses and fluxes in a vertical slot natural convection flow at Rayleigh $R a=10^{5}$ and $5.410^{5}$. Int. J. Heat Fluid Fl., 18(1):70-79, 1997.

[15] T.A.M. Versteegh and F.T.M. Nieuwstadt. A direct numerical simulation of natural convection between two infinite vertical differentially heated walls scaling laws and wall functions. Int. J. Heat Mass Tran., 42(19):3673-3693, 1999.

[16] Joong Hun Bae, Jung Yul Yoo, and Haecheon Choi. Direct numerical simulation of turbulent supercritical flows with heat transfer. Physics of Fluids, 17(10):105104, 2005.

[17] Joong Hun Bae, Jung Yul Yoo, Haecheon Choi, and Donald M. McEligot. Effects of large density variation on strongly heated internal air flows. Physics of Fluids, 18(7):075102, 2006.

[18] S. He, W.S. Kim, and J.H. Bae. Assessment of performance of turbulence models in predicting supercritical pressure heat transfer in a vertical tube. International Journal of Heat and Mass Transfer, 51(19):4659 - 4675, 2008.

[19] S. He, W.S. Kim, and J.D. Jackson. A computational study of convective heat transfer to carbon dioxide at a pressure just above the critical value. Applied Thermal Engineering, 28(13):1662 - 1675, 2008.

[20] P. Kiš and H. Herwig. The near wall physics and wall functions for turbulent natural convection. Int. J. Heat Mass Tran., 55(9-10):2625-2635, 2012. 
[21] Sergio Pirozzoli, Matteo Bernardini, Roberto Verzicco, and Paolo Orlandi. Mixed convection in turbulent channels with unstable stratification. Journal of Fluid Mechanics, 821:482-516, 2017.

[22] Franck Nicoud and Thierry Poinsot. DNS of a channel flow with variable properties. In TSFP DIGITAL LIBRARY ONLINE. Begel House Inc., 1999.

[23] B. Debusschere and C.J. Rutland. Turbulent scalar transport mechanisms in plane channel and Couette flows. International Journal of heat and mass transfer, 47(8):1771-1781, 2004.

[24] Frédéric Auléry, Adrien Toutant, Françoise Bataille, and Ye Zhou. Energy transfer process of anisothermal wallbounded flows. Physics Letters A, 379(24):1520-1526, 2015.

[25] Ashish Patel, Jurriaan W. R. Peeters, Bendiks J. Boersma, and Rene Pecnik. Semi-local scaling and turbulence modulation in variable property turbulent channel flows. Physics of Fluids, 27(9):095101, 2015.

[26] Ashish Patel, Bendiks J. Boersma, and Rene Pecnik. The influence of near-wall density and viscosity gradients on turbulence in channel flows. Journal of Fluid Mechanics, 809:793-820, 2016.

[27] Wen-Ping Wang and Richard H. Pletcher. On the large eddy simulation of a turbulent channel flow with significant heat transfer. Physics of Fluids (1994-present), 8(12):3354-3366, 1996.

[28] Bamdad Lessani and Miltiadis V. Papalexandris. Numerical study of turbulent channel flow with strong temperature gradients. International Journal of Numerical Methods for Heat \& Fluid Flow, 18(3/4):545-556, 2008.

[29] Sylvain Serra, Adrien Toutant, and Françoise Bataille. Thermal large eddy simulation in a very simplified geometry of a solar receiver. Heat Transfer Engineering, 33(6):505-524, 2012.

[30] Syed Mohd Yahya, Syed Fahad Anwer, and Sanjeev Sanghi. Turbulent forced convective flow in an anisothermal channel. International Journal of Thermal Sciences, 88:84-95, 2015.

[31] Guillaume Brillant, Sabine Husson, and Françoise Bataille. Subgrid-scale diffusivity: Wall behavior and dynamic methods. Journal of applied mechanics, 73(3):360-367, 2006.

[32] Sabine Husson. Simulations des grandes échelles pour les écoulements turbulents anisothermes. PhD thesis, Institut National des Sciences Appliquées de Lyon, 2007.

[33] A. Châtelain, F. Ducros, and O. Métais. LES of turbulent heat transfer: proper convection numerical schemes for temperature transport. International Journal for Numerical Methods in Fluids, 44(9):1017-1044, 2004.

[34] W. Sutherland. The viscosity of gases and molecular force. Philosophical Magazine Series 5, 36(223):507-531, 1893.

[35] L. Davidson. Second-order corrections of the $k$ - $\varepsilon$ model to account for non-isotropic effects due to buoyancy. Int. J. Heat Mass Tran., 33(12):2599-2608, 1990.

[36] R.E. Spall, A. Richards, and D.M. McEligot. An assessment of $k-\omega$ and $v^{2}-f$ turbulence models for strongly heated internal gas flows. Numer. Heat Tr. A-Appl., 46(9):831-849, 2004.

[37] R.E. Spall, E. Nisipeanu, and A. Richards. Assessment of a second-moment closure model for strongly heated internal gas flows. J. Heat Transf., 129(12):1719-1722, 2007.

[38] Florian R. Menter. Two-equation eddy-viscosity turbulence models for engineering applications. AIAA journal, 32(8):1598-1605, 1994.

[39] F. Billard and D. Laurence. A robust k- $\varepsilon-/ \mathrm{k}$ elliptic blending turbulence model applied to near-wall, separated and buoyant flows. International Journal of Heat and Fluid Flow, 33(1):45-58, 2012.

[40] Rémi Manceau and Kemal Hanjalić. Elliptic blending model: A new near-wall Reynolds-stress turbulence closure. Physics of Fluids (1994-present), 14(2):744-754, 2002.

[41] R. Manceau. Recent progress in the development of the elliptic blending Reynolds-stress model. Int. J. Heat Fluid Fl., 51:195-220, 2015.

[42] T.B. Gatski and J.-P. Bonnet. Compressibility, Turbulence and High Speed Flow. Academic Press, second edition, 2013. 
[43] F.R. Menter, M. Kuntz, and R. Langtry. Ten years of industrial experience with the SST turbulence model. In Proc. 4th Int. Symp. Turbulence, Heat and Mass Transfer, Antalya, Turkey, 2003.

[44] Paul A. Durbin. Near-wall turbulence closure modeling without "damping functions". Theoretical and Computational Fluid Dynamics, 3(1):1-13, 1991.

[45] S. Parneix, M. Behnia, and P. A. Durbin. Predictions of Turbulent Heat Transfer in an Axisymmetric Jet Impinging on a Heated Pedestal. J. Heat Transf., 120:1-7, 1998.

[46] R. Manceau, S. Parneix, and D. Laurence. Turbulent heat transfer predictions using the $\overline{v^{2}}-f$ model on unstructured meshes. Int. J. Heat Fluid Fl., 21(3):320-328, 2000.

[47] A. Sveningsson and L. Davidson. Computations of flow field and heat transfer in a stator vane passage using the $\overline{v^{2}}-f$ turbulence model. J. Turbomach., 127(3):627-634, 2005.

[48] F. Dehoux, S. Benhamadouche, and R. Manceau. An elliptic blending differential flux model for natural, mixed and forced convection. Int. J. Heat Fluid Fl., 63:190-204, 2017.

[49] Paul A. Durbin. Separated flow computations with the k-epsilon-v-squared model. AIAA journal, 33(4):659-664, 1995.

[50] Charles G. Speziale, Sutanu Sarkar, and Thomas B. Gatski. Modelling the pressure-strain correlation of turbulence: an invariant dynamical systems approach. Journal of Fluid Mechanics, 227:245-272, 1991.

[51] Bart J. Daly and Francis H. Harlow. Transport equations in turbulence. The Physics of Fluids, 13(11):2634-2649, 1970.

[52] B. E. Launder. On the computation of convective heat transfer in complex turbulent flows. Journal of Heat Transfer, 110:1112-1128, 1988.

[53] S. Jakirlić and K. Hanjalić. A new approach to modelling near-wall turbulence energy and stress dissipation. Journal of Fluid Mechanics, 459:139-166, 2002.

[54] Frédéric Archambeau, Namane Méchitoua, and Marc Sakiz. Code saturne: a finite volume code for the computation of turbulent incompressible flows, industrial applications. International Journal on Finite Volumes, 1(1):1-62, 2004.

[55] Sylvain Serra, Adrien Toutant, Françoise Bataille, and Ye Zhou. High-temperature gradient effect on a turbulent channel flow using thermal large-eddy simulation in physical and spectral spaces. Journal of Turbulence, 13:1-25, 2012 .

[56] Franck Nicoud. Numerical study of a channel flow with variable properties. CTR Annual Research Briefs, pages 289-309, 1998.

[57] Bamdad Lessani and Miltiadis V. Papalexandris. Time-accurate calculation of variable density flows with strong temperature gradients and combustion. Journal of Computational Physics, 212:218-246, 2006.

[58] Frederic Aulery, Dorian Dupuy, Adrien Toutant, Françoise Bataille, and Ye Zhou. Spectral analysis of turbulence in anisothermal channel flows. Computers \& Fluids, 151:115-131, 2017. 